\title{
Altered trafficking and stability of polycystins underlie polycystic kidney disease
}

\author{
Yiqiang Cai, ${ }^{1}$ Sorin V. Fedeles, ${ }^{1}$ Ke Dong, ${ }^{1}$ Georgia Anyatonwu, ${ }^{2}$ Tamehito Onoe, ${ }^{1}$ Michihiro Mitobe, ${ }^{1}$ Jian-Dong Gao, ${ }^{1}$ \\ Dayne Okuhara, ${ }^{1}$ Xin Tian, ${ }^{1}$ Anna-Rachel Gallagher, ${ }^{1}$ Zhangui Tang, ${ }^{1}$ Xiaoli Xie, ${ }^{1}$ Maria D. Lalioti, ${ }^{3}$ Ann-Hwee Lee, ${ }^{4}$ \\ Barbara E. Ehrlich, ${ }^{2}$ and Stefan Somlo ${ }^{1,5}$
}

'Department of Internal Medicine, 'Department of Pharmacology, and ${ }^{3}$ Department of Obstetrics, Gynecology and Reproductive Science, Yale University School of Medicine, New Haven, Connecticut, USA. ${ }^{4}$ Department of Pathology and Laboratory Medicine, Weill Cornell Medical College, New York, New York, USA. ${ }^{5}$ Department of Cenetics, Yale University School of Medicine, New Haven, Connecticut, USA.

\begin{abstract}
The most severe form of autosomal dominant polycystic kidney disease occurs in patients with mutations in the gene (PKD1) encoding polycystin-1 (PC1). PC1 is a complex polytopic membrane protein expressed in cilia that undergoes autoproteolytic cleavage at a G protein-coupled receptor proteolytic site (GPS). A quarter of PKD1 mutations are missense variants, though it is not clear how these mutations promote disease. Here, we established a cell-based system to evaluate these mutations and determined that GPS cleavage is required for PC1 trafficking to cilia. A common feature among a subset of pathogenic missense mutations is a resulting failure of PC1 to traffic to cilia regardless of GPS cleavage. The application of our system also identified a missense mutation in the gene encoding polycystin-2 (PC2) that prevented this protein from properly trafficking to cilia. Using a Pkd1-BAC recombineering approach, we developed murine models to study the effects of these mutations and confirmed that only the cleaved form of PC1 exits the ER and can rescue the embryonically lethal Pkd1-null mutation. Additionally, steady-state expression levels of the intramembranous $\mathrm{COOH}$-terminal fragment of cleaved PC1 required an intact interaction with PC2. The results of this study demonstrate that PC1 trafficking and expression require GPS cleavage and PC2 interaction, respectively, and provide a framework for functional assays to categorize the effects of missense mutations in polycystins.
\end{abstract}

\section{Introduction}

The most common and severe form of autosomal dominant polycystic kidney disease (ADPKD) results from mutations in PKD1. PKD1 encodes human polycystin-1 (PC1), a large, complex, lowabundance polytopic membrane protein with structural features suggestive of a cell-surface receptor (1). PC1 is the common rate-limiting component of the ADPKD signaling pathway, and functional dosage of the gene also modulates the phenotypes in isolated autosomal dominant polycystic liver disease and autosomal recessive polycystic kidney disease (2). Mutations in PKD2, encoding human polycystin-2 (PC2), cause ADPKD with an identical, albeit milder, phenotypic spectrum compared with that of PKD1. PC2 is a member of the transient receptor potential (TRP) family of cation channels. Biochemical evidence shows that PC1 and PC2 interact directly $(3,4)$, and genetic and phenotypic evidence suggests that they act in a common pathway $(2,5-7)$. The prevailing hypothesis is that PC1 and PC2 form a receptorchannel complex with either mechanical or ligand stimuli as the upstream signals (8-11), although direct evidence for this functional interrelationship is lacking. Coassembly with PC1 has been proposed as a requirement for trafficking of PC2 to the cell surface (12), although PC2 can traffic to cilia independently of PC1 (13). There is also evidence for divergent functions for PC1 and PC2: e.g., PC2 is involved in left-right axis determination (14-16) and

Conflict of interest: The authors have declared that no conflict of interest exists Submitted: April 18, 2014; Accepted: September 30, 2014.

Reference information: J Clin Invest. 2014;124(12):5129-5144. doi:10.1172/JCI67273.
$\mathrm{Ca}^{2+}$ homeostasis in the ER $(17,18)$, whereas PC1 can be expressed in association with the lateral membrane (19-21), adherens junctions (22), and desmosomes (23).

Several lines of evidence have led to the emergence of the primary cilium as the central cellular compartment in the pathogenesis of cystic kidney diseases (24). PC1 and PC2 are expressed in the membrane overlying the primary cilium $(25,26)$. The majority of gene products associated with fibrocystic kidney diseases other than ADPKD are also expressed in the cilia-basal body complex $(24,27)$. A forward genetic screen in zebrafish based on the cystic pronephros phenotype identified a number of cilia-related proteins as well as PC2 (28). Fibrocystic disease-associated gene products, including PC2, are associated with other known cilial phenotypes such as left-right axis defects (14-16). In addition, the role of cilia in cyst formation was tested prospectively by showing that disruption of cilia through selective inactivation of Kif3a or intraflagellar transport proteins in the kidney results in cyst formation (29-31). Recently, we have found that cyst formation following inactivation of PC1 or PC2 is markedly slowed by concomitant removal of structurally intact cilia, suggesting that intact cilia devoid of polycystins are the primary driving force in the progression of ADPKD (32). Despite these cogent arguments, direct evidence for the precise mechanism of PC1 and PC2 action in cilia has been elusive.

Human PC1 is composed of 4,302 amino acids with approximately 3,000 amino acid extracellular $\mathrm{NH}_{2}$ termini, 11 transmembrane domains (33), and approximately 220 amino acid cytosolic $\mathrm{COOH}$ termini. The extracellular $\mathrm{NH}_{2}$-terminal domain contains a distinct combination of protein motifs including immunoglobulin- 
like PKD repeats (34-36), a receptor egg jelly (REJ) domain (37) that includes fibronectin type III repeats (38) and is part of a recently identified structural GAIN domain (39). The REJ/GAIN domain is required for autoproteolytic cleavage of $\mathrm{PC} 1$ at a $\mathrm{G}$ protein-coupled receptor proteolytic site (GPS) sequence, $H L \downarrow T^{3049}$ (40), that yields an extracellular $\mathrm{NH}_{2}$-terminal fragment (PC1-NTF) and an intramembranous COOH-terminal fragment (PC1-CTF) (39, 41, 42). PC1-NTF and PC1-CTF remain noncovalently associated with each other. The conservation of the GPS in all PC1 homologs (43) suggests functional importance; although at least 2 homologs, PKDREJ and SuREJ2, do not undergo GPS cleavage $(44,45)$.

Cysts in ADPKD are thought to occur primarily by a cellular recessive 2-hit mechanism, with the majority of clinically significant heterozygous germline mutations in PKD1 and PKD2 resulting in loss of function. Nonetheless, approximately $25 \%$ of the presumed pathogenic variants in PC1 are predicted to result in nonsynonymous amino acid substitution mutations (46). Increasing evidence shows that a significant proportion of these nonsynonymous substitution variants are functionally hypomorphic and have a milder clinical presentation compared with that of complete loss-of-function variants $(47,48)$. The pathogenic effects of such variants have proven difficult to assess experimentally in the absence of robust functional assays for PC1. In the current study, we examined the functional expression and cilia-trafficking properties of mutant forms of murine PC1 and human PC2 in vitro and of PC1 in vivo. We found that a subset of pathogenic amino acid substitution mutations in PC1 and PC2, as well as mutations affecting GPS cleavage in PC1, result in failure of polycystins to traffic to cilia. To extend these cell-based studies to mammalian tissues in vivo, we modified a $P k d 1 \mathrm{BAC}$ transgene that can fully rescue the embryonically lethal $P k d 1^{-/-}$phenotype with a mutation to abrogate GPS cleavage and showed that this mutation results in a complete loss-of-function allele that can no longer rescue any part of the $\mathrm{Pkd1}^{-/-}$phenotype. We used the BAC transgenic models to establish that there are significant differences in the subcellular compartment distributions of PC1 and murine PC2 in tissues, supporting the hypothesis that these proteins may also have functions independent of each other. Finally, we examined PC1 expression in the absence of PC2 and found that interaction with PC2 is required to maintain steady-state expression levels of PC1-CTF. These studies highlight the role of defective polycystin trafficking and expression in all forms of ADPKD and suggest a role for therapies directed at these processes in a selected subset of ADPKD patients.

\section{Results}

Asparagine-linked glycosylation and apical expression of PC1. Fulllength mouse PC1 (PC1-FL) with $\mathrm{NH}_{2}^{-}$and $\mathrm{COOH}$-terminal epitope tags (49) cleaved at the GPS site to yield the HA epitopetagged PC1-CTF and the FLAG epitope-tagged PC1-NTF, which remained noncovalently associated with each other (ref. 41 and Figure 1A). We examined the asparagine-linked glycosylation ( $N$-glycosylation) pattern of PC1. PC1-CTF showed both endoglycosidase $\mathrm{H}$-resistant (endo $\mathrm{H}$-resistant) and -sensitive forms, whereas the full-length, uncleaved PC1 protein PC1-FL was entirely endo $\mathrm{H}$ sensitive (Figure 1, B and C, and ref. 50). Endo $\mathrm{H}$ resistance defines the fraction of $\mathrm{PC1}-\mathrm{CTF}$ that traffics past the middle Golgi apparatus to the cell surface. Digestion with pep- tide- $N$-glycosidase F (PNGase F) removes all $N$-glycan moieties. An absence of endo $\mathrm{H}$ resistance in PC1-FL shows that uncleaved PC1 is not present on the cell surface. Anti-FLAG immunoprecipitation (IP) of PC1-NTF only coimmunoprecipitated the intracellular endo $\mathrm{H}-$ sensitive fraction of PC1-CTF (Figure 1C). The failure to detect the cell-surface pool of PC1-NTF was likely due to shedding of the surface-expressed PC1-NTF into the culture medium (ref. 41 and Figure 1D). We further examined the surface location of PC1 biochemically by selective biotinylation of the apical and basolateral

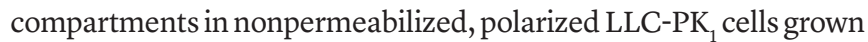
on filter inserts (51). We found that PC1-CTF was strongly biotinylated on the apical surface of cells, but was virtually absent from the basolateral surface (Figure 1E). Uncleaved PC1-FL was not detected by cell-surface biotinylation in live cells (Figure 1E). We next determined whether the absence of PC1-FL from the cell surface was indicative of very efficient cleavage of PC1 during trafficking or an intrinsic property of uncleaved PC1. The cleavage-deficient mutant $\mathrm{PC}^{\mathrm{L} 304 \mathrm{OH}}$ bearing an amino acid substitution at the consensus GPS $\mathrm{HL}_{3040} \downarrow \mathrm{T}$ of the mouse sequence remained completely endo $\mathrm{H}$ sensitive, indicating that the uncleaved protein cannot reach the cell surface (Figure 1F). The fact that PC1-CTF acquired endo $\mathrm{H}$ resistance suggests that its path to the cell surface passes through the Golgi apparatus. The biotinylation pattern in polarized epithelial cells shows that in the steady state, PC1-CTF is preferentially expressed on the apical surface in at least some cell types.

Truncation mutations and cilia trafficking of PC1. The site of expression of PC1 on the apical surface most relevant to the pathogenesis of ADPKD is the primary cilium. We investigated the structural elements of PC1 that are critical to cilia trafficking. We hypothesized that defective trafficking of PC1 to cilia would serve as an in vitro functional test for a subset of putative pathogenic variants in Pkd1. For the purposes of this study, all human PC1 sequence variants were introduced into the equivalent codon in mouse $P k d 1$ cDNA to achieve the same amino acid substitution. The variants are described using mouse sequence nomenclature and mouse-to-human amino acid equivalencies, and the pathogenic potential for all variants are presented in Supplemental Table 1 (supplemental material available online with this article; doi:10.1172/JCI67273DS1). PC1 was detected in cilia by immunofluorescence (IF) cell staining using $\mathrm{COOH}$ - or $\mathrm{NH}_{2}$-terminal epitopes independently in a variety of cell lines including previously reported mouse embryonic fibroblast (MEFs) (2), LLC-PK cells (Figure 2A), and IMCD3 and MDCK cells (data not shown). The HA and FLAG epitopes colocalized in cilia, indicating that both PC1-CTF and PC1-NTF appeared in cilia together (Figure 2A). We observed PC1 expression along the entire axoneme of the cilia, and PC1 did not colocalize with basal bodies marked by $\gamma$-tubulin (Figure 2, A and B). The cleavage-deficient $\mathrm{PC}^{\mathrm{L} 3040 \mathrm{H}}$ was not detectable in cilia axonemes or at the basal bodies despite robust expression in the cell body, showing that GPS cleavage-deficient PC1 carrying this specific GPS motif variant did not traffic to cilia (Figure 2C). These findings imply that the HA and FLAG epitopes are detecting cleaved PC1-CTF and PC1-NTF, respectively, as the only forms of PC1 in cilia.

Between $65 \%$ and $75 \%$ of pathogenic mutations in PKD1 are either nonsense, splice site, or insertion/deletion changes that are predicted to result in premature termination of the PC1 pep- 
A

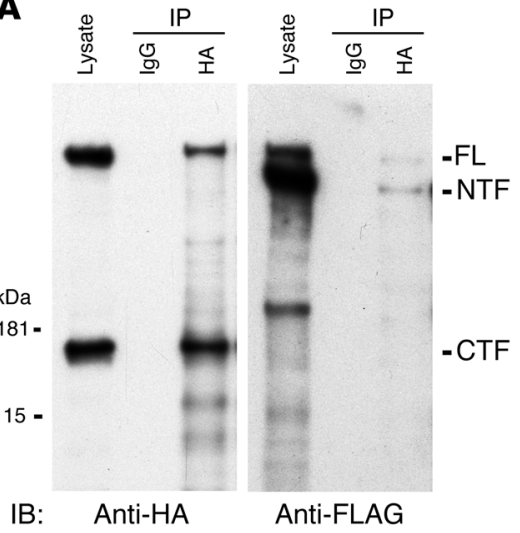

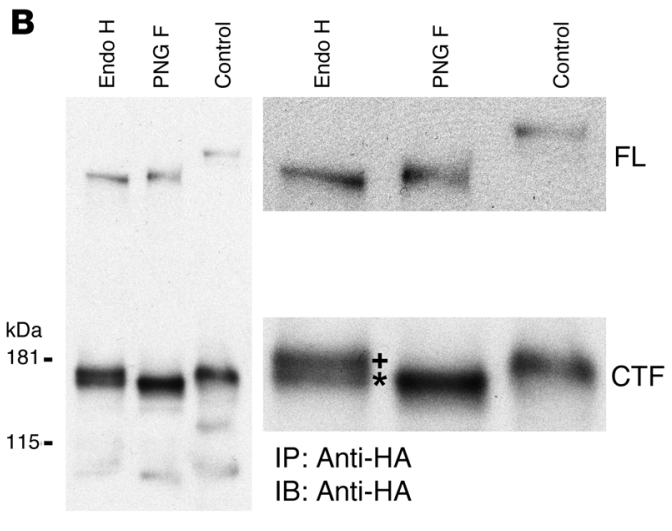

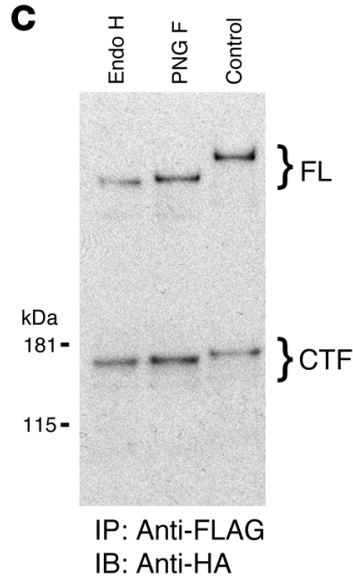

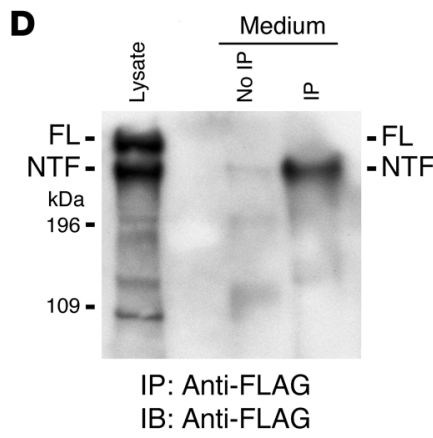
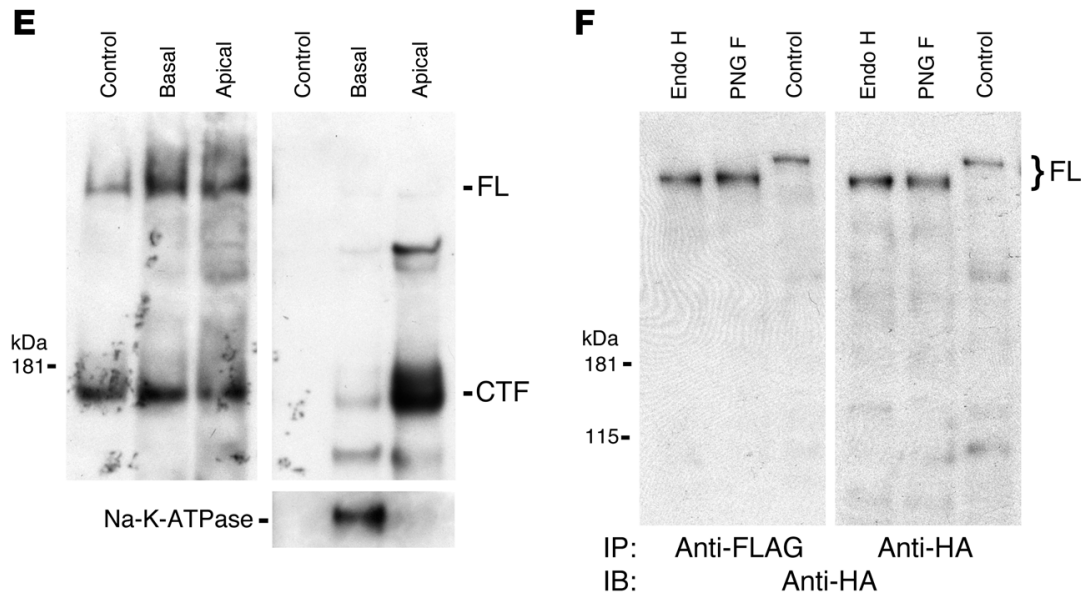

Figure 1. $\mathbf{N}$-glycosylation and surface expression of PC1. (A) LLC-PK cell lysates expressing PC1 with $\mathrm{NH}_{2}$-terminal triple-FLAC and COOH-terminal triple-HA epitope tags underwent IP with either nonimmune IgG (IgG) or anti-HA (HA) and IB with anti-HA (left) and anti-FLAG (right). The 2 panels are from a single gel and show PC1-NTF (NTF), PC1-CTF (CTF), and uncleaved full-length PC1 (FL). (B) PC1 IP followed by digestion with endo H, PNGase F (PNG F), or reaction buffer (Control). Panels at right are magnifications of the left panel (original magnification, $\times 2$ ). PC1-CTF shows endo H-resistant $(+)$ and -sensitive $\left(^{*}\right)$ products. PC1-FL shows complete endo H sensitivity. (C) IP of PC1 by anti-FLAG coimmunoprecipitated the endo H-sensitive fraction of PC1-CTF, indicative of the intracellular PC1-NTF/PC1-CTF pool. (D) Shedding of PC1-NTF into the culture medium may explain the absence of a detectable cell-surface PC1-NTF/PC1-CTF complex. Medium was either run directly (No IP) or subjected to IP prior to IB. Lysate, total cell lysate. (E) LLC-PK cells grown on semipermeable supports selectively labeled with biotin on the apical or basal surface. Total lysates (left panel) and streptavidin-precipitated biotinylated proteins (right panel) were run on a single gel and analyzed by IB with anti-HA (upper 2 panels) and anti-Na-K-ATPase (lower panel). PC1-CTF was biotinylated on the apical surface. Na-K-ATPase served as a positive control for the basal surface. (F) GPS cleavage-deficient PC1 ${ }^{13040 \mathrm{H}}$ was entirely endo $\mathrm{H}$ sensitive, suggesting that it did not traffic past the middle Golgi apparatus in cells; the 2 panels show noncontiguous lanes on a single gel.

tide chain $(46,48)$. We determined the effect of such mutations in cilia trafficking of PC1. We selected the naturally occurring human mutation $\mathrm{PC} 1^{\mathrm{R} 4228 \mathrm{X}}$ (mouse equivalent, $\mathrm{PC} 1^{\mathrm{R} 4218 \mathrm{X}}$ ), which has been shown to abrogate interaction with PC2 (12). In addition, we engineered 2 experimental truncations, $\mathrm{PC}^{\mathrm{R} 4204 \mathrm{X}}$ and $\mathrm{PC} 1^{\mathrm{Y} 4100 \mathrm{x}}$, based on the mouse sequence. The former truncates PC1 before the coiled-coil domain required for interaction with PC2 $(52,53)$; the latter deletes the entire predicted cytoplasmic $\mathrm{COOH}$ terminus (33). All 3 truncation mutants showed normal cleavage at the GPS when stably expressed in LLC-PK ${ }_{1}$ epithelial cells (Figure 2D). The $\mathrm{PC}^{\mathrm{R} 4218 \mathrm{X}}$ mouse equivalent of the human pathogenic mutant was detectable in cilia by both the $\mathrm{COOH}$-terminal HA epitope and the $\mathrm{NH}_{2}$-terminal FLAG epitope (Figure 2, E and F). We confirmed the specificity of these findings by colabeling with $\gamma$-tubulin to detect basal bodies and avoid possible cross-reactivity along the axoneme (Figure 2, G and $\mathrm{H}$ ). $\mathrm{PC}^{\mathrm{R} 4204 \mathrm{x}}$ similarly appeared in cilia by both HA and FLAG epitope detection (Figure 2, I and J). The truncation mutant lacking the entire $\mathrm{COOH}$ terminus $\mathrm{PC}^{\mathrm{Y}}{ }^{4100 \mathrm{x}}$ failed to reach cilia when detected by either epitope (Figure 2, K and L) despite stable expression of the protein product and GPS cleavage (Figure 2D). In keeping with this failure to traffic to cilia, $\mathrm{PC1}^{\mathrm{Y} 4100 \mathrm{X}_{-}}$ CTF did not acquire endo $\mathrm{H}$ resistance (Figure 2D). These data indicate that the region between $\mathrm{Y} 4100$ and R4204 is necessary for trafficking of PC1 to cilia, that interaction with PC2 is not required for GPS cleavage or cilia trafficking of PC1, and that GPS cleavage is not sufficient for cilia trafficking of PC1.

Missense mutations and cilia trafficking of PC1. The remaining $25 \%$ to $35 \%$ of pathogenic PKD1 mutations are predicted to result in nonsynonymous amino acid substitutions. To date, there have been no systematic in vitro assays to assess the pathogenicity of these variants. This has made the functional assessment of disease-causing missense mutations challenging. We evalu- 

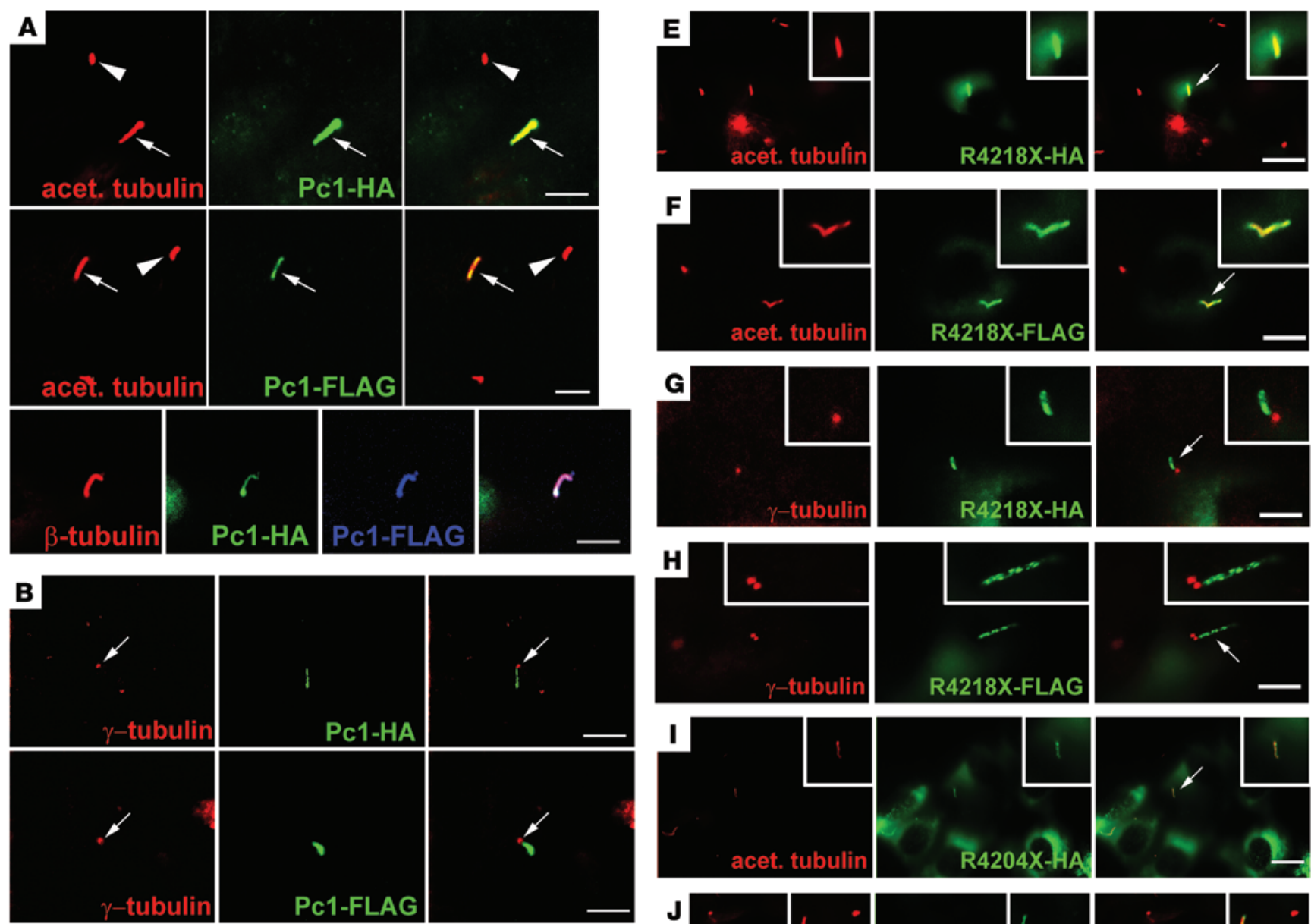

R4218X-FLAG

$-$
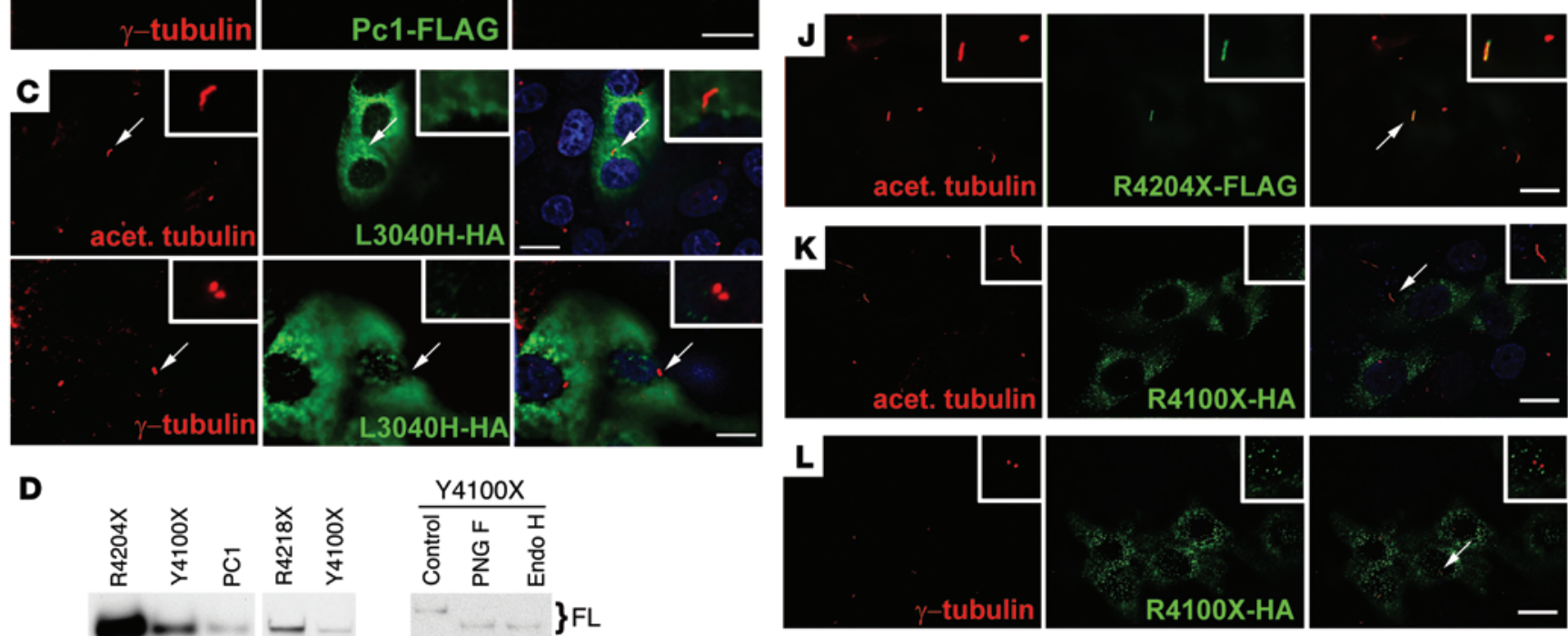

R4204X-AA

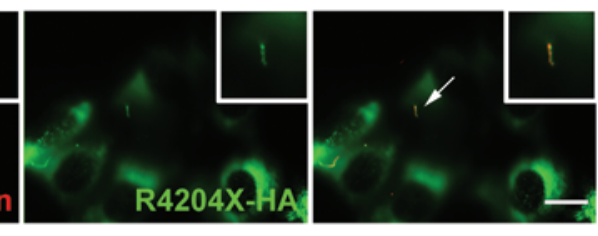

D
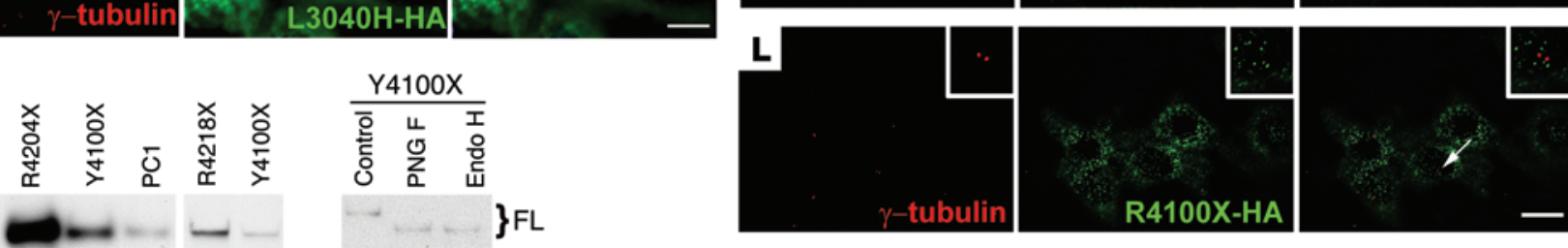

$\mathrm{kDa}$

181

$+\cdots+$ \}CTF 
Figure 2. Expression of PC1 in cilia. (A) PC1 expression detected by the $\mathrm{COOH}$-terminal $\mathrm{HA}$ epitope and $\mathrm{NH}_{2}$-terminal FLAG epitope in cilia (arrows) of LLC-PK ${ }_{1}$ cells. Bottom row of panels show triple labeling of a single cell using rabbit anti-FLAG and preconjugated mouse anti- $\beta$-tubulin and anti-HA antibodies. Merged images are shown at right. (B) PC1 did not colocalize with basal bodies, marked by $\gamma$-tubulin (arrows). (C) PC1 $1^{\mathrm{L3040H}}$ expressed in LLC-PK ${ }_{1}$ cells did not traffic to cilia. Inserts are $\times 2$ magnified reconstructions from image stacks used to verify that cell bodies belonging to the respective cilia and basal bodies were expressing PC1 ${ }^{13040 \mathrm{H}}$. (D) CPS cleavage was normal in the truncation mutants of PC1, as shown by IB with anti-HA (left panel). TPC $1^{14100 x}$-CTF showed no endo-H-resistant species (right panel). The 3 panels were run on separate gels. (E-L) Cilia trafficking of truncated forms of PC1. (E-H) The PC1 ${ }^{\text {R4218X }}$ variant orthologous to the $P C 1^{14228 x}$ human mutation and (I and $\mathrm{J}$ ) the $\mathrm{PC} 1^{\mathrm{R} 4204 \mathrm{X}}$ truncation placed before the coiled-coil domain were both expressed in cilia. ( $K$ and L) $P C 1^{R 4100 x}$, truncating the entire $\mathrm{COOH}$ terminus, was not expressed in cilia. The $\mathrm{COOH}$ terminus of PC1 was detected with anti-HA (E, G, I, K, and $\mathbf{L})$, and the $\mathrm{NH}_{2}$ terminus was detected with anti-FLAC $(\mathbf{F}, \mathbf{H}$, and $\mathbf{J})$. Cilia axonemes were marked by anti-acetylated tubulin (E, F, I, J, and $\mathbf{K})$, and basal bodies were marked by anti- $\gamma$-tubulin $(\mathbf{G}, \mathbf{H}$, and $\mathbf{L}$ ). The rightmost panel for each series of images in $\mathbf{A}-\mathbf{C}$ and $\mathbf{E}-\mathbf{L}$ represents a merge of the images at left. Scale bars: $5 \mu \mathrm{m}$.

ated a series of human pathogenic missense variants in an effort to categorize their functional consequences by in vitro assays of cilia trafficking. We first determined whether naturally occurring mutations that impair GPS cleavage also abrogate cilia trafficking of PC1. A previous study had shown that 2 human mutations remote from the actual $G$ protein-coupled proteolytic cleavage sites, $\mathrm{PC}^{\mathrm{E} 2771 \mathrm{~K}}$ and $\mathrm{PC} \mathrm{1}^{\mathrm{F} 2853 \mathrm{~S}}$, respectively corresponding to mouse codons $\mathrm{PC}^{\mathrm{E} 2766}$ and $\mathrm{PC}^{\mathrm{F} 2848}$, abrogate GPS cleavage (41). These mutations, originally referred to as REJ mutations, occur in the GAIN domain recently defined by structural studies as being required for autoproteolytic cleavage at the GPS (39). For the current study, we selected another human mutation, PC1 ${ }^{\text {L2816P }}$, corresponding to mouse PC1 ${ }^{\text {L2811P }}$, which is located in the GAIN domain between $\mathrm{PC}^{\mathrm{E} 2271 \mathrm{~K}}$ and $\mathrm{PC} 1^{\mathrm{F} 2853 \mathrm{~S}}$ and is considered likely to be pathogenic (Supplemental Table 1). PC1 ${ }^{\mathrm{L} 2811 \mathrm{P}}$ did not cleave at the GPS (Figure 3A) and did not localize to cilia (Figure 3B). As a positive control for the assay technique, the variant $\mathrm{PC}^{\mathrm{C} 2085 \mathrm{R}}$ that changes a nonconserved residue in the fifteenth PKD domain in the mouse sequence ( $\left.\mathrm{PC}^{\mathrm{C} 2085}\right)$ to the equivalent amino acid from the human sequence $\left(\mathrm{PC}^{\mathrm{R} 2089}\right)$ was cleaved normally at the GPS (Figure 3A) and trafficked normally to cilia (Figure 3C). The finding that 2 independent mutations, $\mathrm{PC} 1^{\mathrm{L} 3040 \mathrm{H}}$ and $\mathrm{PC} 1^{\mathrm{L} 2811 \mathrm{P}}$, which result in loss of GPS cleavage, also failed to traffic to cilia is consistent with the hypothesis that GPS cleavage is required for trafficking of PC1 to cilia.

We next investigated whether cilia-trafficking defects were a feature of amino acid substitution mutations in the extracellular domain of PC1, irrespective of their effects on GPS cleavage. We selected a pair of human mutations, $\mathrm{PC} 1^{\mathrm{C} 210 \mathrm{G}}$ and $\mathrm{PC} 1^{\mathrm{V} 690 \mathrm{D}}$, outside the GAIN domain region that were predicted to be pathogenic (Supplemental Table 1). PC1 ${ }^{\mathrm{C} 210 \mathrm{G}}$ alters a highly conserved cysteine in the WSC domain. $\mathrm{PC}^{1690 \mathrm{D}}$ is in the interdomain region between the $\mathrm{C}$-lectin and PKD domains. We produced the corresponding mouse variants, $\mathrm{PC}^{\mathrm{C} 210 \mathrm{G}}$ and $\mathrm{PC} 1^{\mathrm{V} 685 \mathrm{D}}$. $\mathrm{PC}^{\mathrm{C} 210 \mathrm{G}}$ and $\mathrm{PC}^{\mathrm{V} 685 \mathrm{D}}$ cleaved normally at the GPS (Figure 3A). Despite normal GPS cleavage and robust expression in the cell body, $\mathrm{PC}^{\mathrm{C} 210 \mathrm{G}}$ and $\mathrm{PC}^{\mathrm{V} 685 \mathrm{D}}$ failed to traffic to cilia (Figure 3, D and E, and Supplemental Figure 1). These data show that pathogenic amino acid substitution mutations in PC1 can abrogate cilia trafficking independently of GPS cleavage and support the hypothesis that cilia trafficking is an effective in vitro surrogate assay for a subset of pathogenic PC1 mutations.

We next determined whether amino acid substitution mutations affect other functions of PC1 besides GPS cleavage and trafficking to cilia. PKD domains were first identified in the extracellular region of PC1 (34-36) and were proposed to have possible ligand-binding functions (54). The PKD domains have a $\beta$-sandwich fold, also called an Ig-like fold, among which a WDFGDGS motif is the most conserved sequence (54). We examined a putative "likely" pathogenic human mutation, PC1 ${ }^{\mathrm{G} 1166 \mathrm{~S}}$ (Supplemental Table 1), that affected the WDFGDG ${ }^{1166} S$ motif in the fifth predicted PKD domain (54). The mouse-equivalent PC1 ${ }^{\mathrm{G1160s}}$ mutant is a stable protein product that was cleaved at the GPS (Figure 3A) and was expressed abundantly in cilia (Figure 3F). If this variant is indeed pathogenic, as suggested by sequence conservation and structural data (54), these findings suggest that functions other than GPS cleavage and cilia trafficking, perhaps involving ligand interactions, may also be disrupted in pathogenic amino acid substitution mutations in PC1.

Missense mutations and cilia trafficking of PC2. Although missense variants are less common in PKD2 ( $6 \%-14 \%)$, we took this opportunity to evaluate whether cilia-trafficking mutations also play a role in ADPKD resulting from mutations in PKD2. We evaluated $\mathrm{PC} 2^{\mathrm{W} 414 \mathrm{G}}$, a mutation in the highly conserved polycystin domain (55) common to both PC1 and PC2 and located in the large extracellular loop between the first and second transmembrane segments of PC2 and the sixth and seventh transmembrane segments of PC1 (43). Single-channel recordings using bilayers made with ER-enriched vesicles from LLC-PK ${ }_{1}$ cells stably expressing $\mathrm{PC} 2{ }^{\mathrm{W} 414 \mathrm{G}}$ showed that the mutation did not abrogate channel activity of PC2 in the ER (Figure 4, A and B). The calcium concentration on the cytoplasmic side needed to maintain the open probability near approximately $50 \%$ was the same for both the WT and $\mathrm{PC} 2^{\mathrm{W} 414 \mathrm{G}}$ channel, suggesting that there were no obvious differences in the open probability between the WT and mutant channels. At higher holding potentials, the currents through $\mathrm{PC}^{\mathrm{W} 414 \mathrm{G}}$ showed a lower conductance than did the currents through the WT channel, but at the lower holding potentials, which were more representative of the ER membrane, the channel conductances were similar for the WT and $\mathrm{PC}^{\mathrm{W} 414 \mathrm{G}}$ mutant. In aggregate, it is unlikely that the differences in channel activity account for the pathogenicity of $\mathrm{PC} 2^{\mathrm{W} 414 \mathrm{G}}$.

$\mathrm{PC} 2^{\mathrm{W} 414 \mathrm{G}}$ did not traffic to cilia despite robust expression in cells, suggesting this to be the more likely mechanism for its pathogenesis (Figure 4C). As a positive control, a channel-dead pathogenic missense variant, $\operatorname{PC}^{\text {D511v }}(17,56)$, trafficked to cilia in the same cell type (Figure 4D). We further confirmed the specificity of the cilia-trafficking defect in $\mathrm{PC} 2^{\mathrm{W} 414 \mathrm{G}}$ by introducing the mutation into the truncated $\mathrm{PC} 2^{\mathrm{L} 703 \mathrm{X}}$ backbone that normally shows particularly robust trafficking to cilia (Figure $4 \mathrm{E}$ and ref. 13). The truncated protein containing the point mutation $\mathrm{PC} 2^{\mathrm{W} 414 \mathrm{G} ; \mathrm{L} 703 \mathrm{X}}$ did not traffic to cilia (Figure 4F). The data suggest that cilia trafficking mutants occur in PC2, that the conserved extracellular domain unique to polycystins is important in trafficking of PC2, 
A
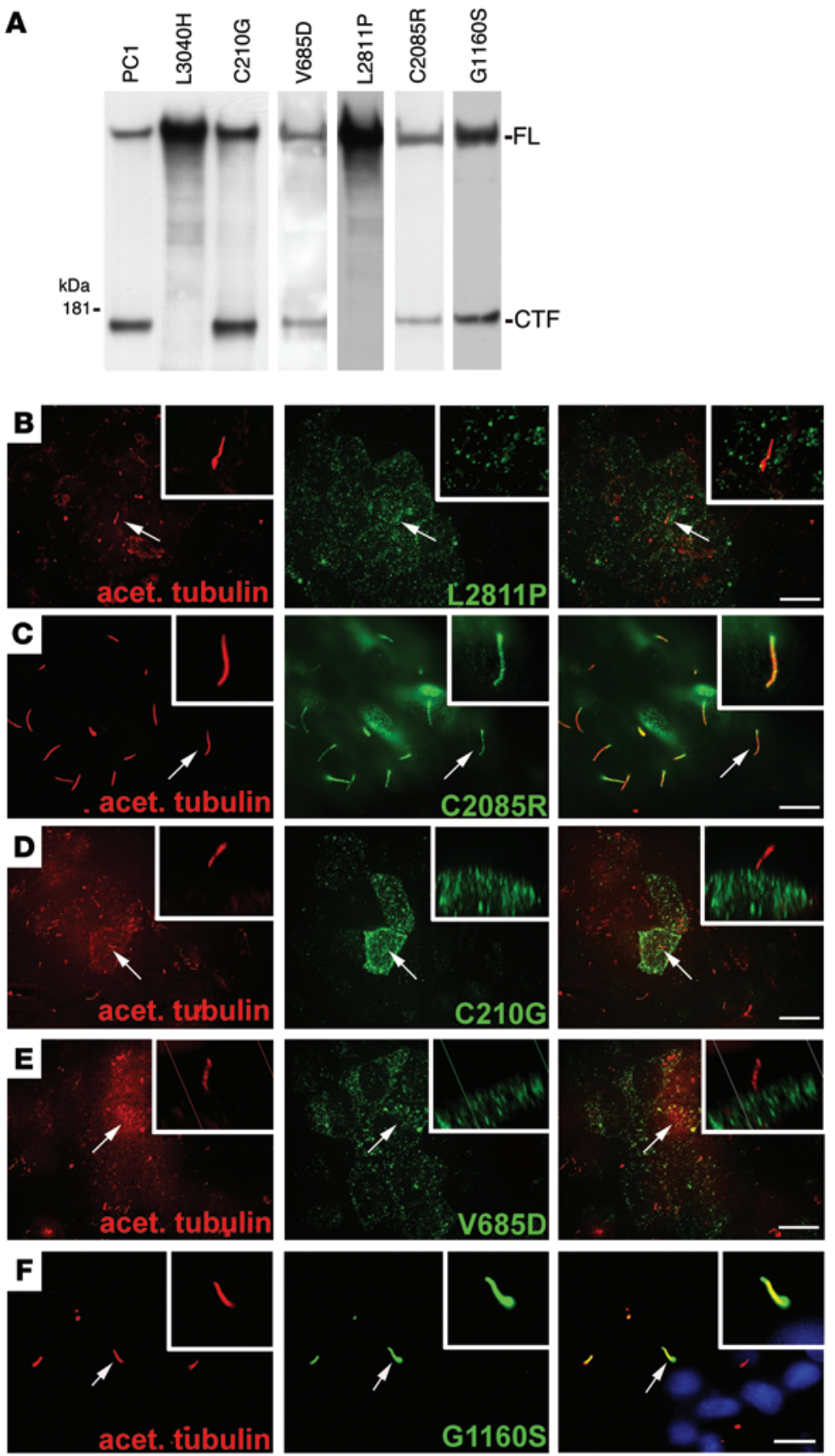

Figure 3. Functional consequences of human pathogenic variants of PC1. (A) Missense variants outside of the predicted GAIN/REJ domain (PC1C ${ }^{210 G}, P C 1^{\mathrm{V685D}}, P C 1^{61160 S}$ ) did not affect GPS cleavage of PC1. The GAIN/REJ mutant PC1 ${ }^{\text {L2811P }}$ abrogated CPS cleavage, whereas the benign polymorphism $\mathrm{PC1}^{\mathrm{C2085R}}$ had no effect. Cell lysates were subjected to IP and IB with anti-HA. Each panel was run on a separate gel. (B-F) Cilia trafficking of the PC1 variants in A. Pathogenic mutants in the REJ/CAIN domain (B) or $\mathrm{NH}_{2}$ terminus ( $\mathbf{D}$ and $\mathbf{E}$ ) of PC1 did not traffic to cilia, whereas a pathogenic mutant in the fifth PKD domain (F) trafficked to cilia similarly to WT protein. (C) The nonpathogenic variant was expressed in cilia. PC1 variants were expressed in LLC-PK cells and IF performed using anti-acetylated $\alpha$-tubulin (red) and anti-FLAG (green) antibodies. Arrows show cilia in $x-y$ images; inserts are $3 \mathrm{D}$ reconstructions from z-stacks that have been rotated to show cilia above the plane of the cell bodies and were used to verify that cell bodies belonging to the respective cilia were expressing PC1 variants (green). Original magnification (inserts), $\times 2$. The rightmost panel for each series of images B-F represents a merge of the images at left. Scale bars: $5 \mu \mathrm{m}$.

models, while still providing high fidelity to native gene expression patterns in a copy number-dependent, integration site-independent manner. We initially modified the mouse BAC clone RPCI22-287A3 containing $P k d 1$ by inserting a triple-FLAG epitope after the $\mathrm{NH}_{2}$-terminal leader sequence and a triple-HA epitope before the stop codon in the same locations as in the Pkd1 cDNA $(2,49)$. BAC clone RPCI22-287A3 contains 7 other genes in their entirety as well as parts of 2 additional genes (Supplemental Table 2). Seven transgenic founder lines produced with this modified $P k d 1^{F / H}-B A C$ transgene gave germline transmission; 4 of these lines were analyzed further (Figure 5 and Supplemental Tables 3 and 4).

BACs typically yield low copy number transgenic insertions, and our founders had 1-8 copies of the $B A C$ transgene as determined by quantitative PCR (qPCR) (Supplemental Table 3). We found that PC1 protein expression determined by semiquantitative immunoblotting (IB) was approximately 2- to 4-fold higher relative to PC1 expressed in the single-copy founder Tg14 (Figure 5, A and B, and Supplemental Table 3). PC1 expression correlated with the transgene copy number in 3 of the 4 founders. It is likely that the 8 "copies" of the BAC detected by qPCR in $\mathrm{Tg} 8$ included some BAC inserts that were disrupted in the integration process, so that only a subset of the BAC inserts actually expressed PC1. The distinct nature of the respective modifications in the $P k d 1^{F / H}-B A C$ transgene and the native $P k d 1$-null allele enabled us to distinguish null, WT, and $B A C$ alleles for purposes of genotyping (Supplemental Figure 2). All 4 founder lines were able to rescue the embryonic lethality when combined with the $\mathrm{Pkd1}^{-1-}$ background (Supplemental Table 4 and ref. 2), indicating that the $B A C$ transgene was effective in producing a functional protein independently of the integration site and copy number and that the epitope tags did not impact in vivo function of PC1. High-copy number $P k d 1$ transgenes have been associated with kidney cyst formation $(60,61)$. We did not observe cyst formation in our low-copy number BAC transgenic models, nor were any other abnormal phe- 
A

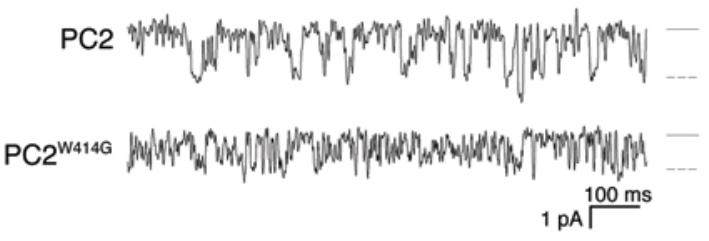

B
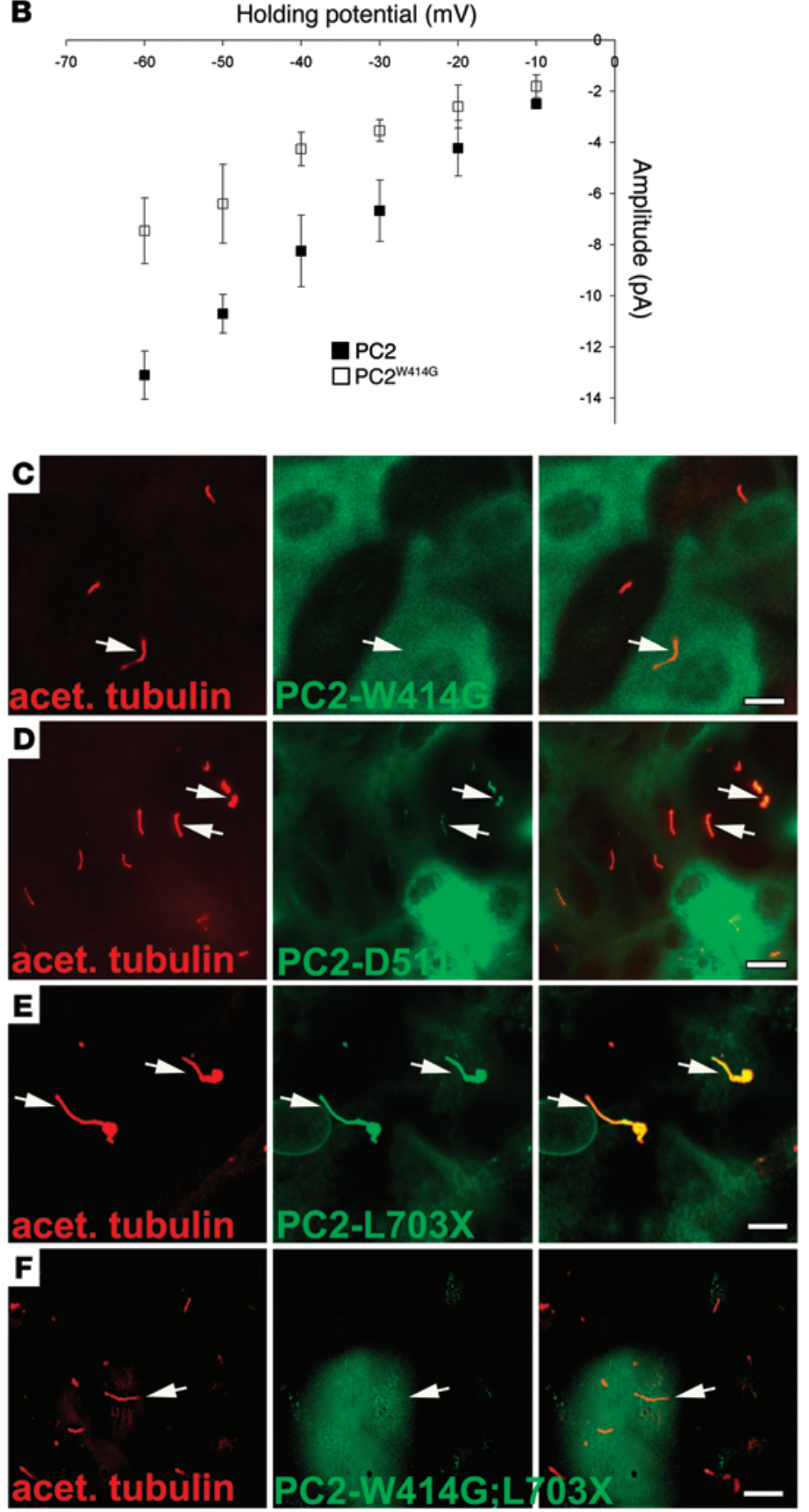

notypes observed in tissue surveys up to 12 months (Supplemental Figure 3, data not shown, and ref. 2).

The $P k d 1^{F / H}-B A C$ transgene expressed readily detectable epitope-tagged PC1 in kidney tissue (Figure 5, A and B, and ref. 2). We observed that the PC1-CTF appeared as 2 bands of differing migration (Figure $5 \mathrm{C}$ ), consistent with the previously reported alternative splicing of exon 31 in the rodent, but not human, tran-
Figure 4. PC2 cilia-trafficking mutations. (A and B) PC2 ${ }^{W 414 C}$ retained channel activity comparable to that of WT PC2. (A) Electrophysiological experiments were performed under asymmetrical conditions (500 $\mathrm{mM} \mathrm{CsCl}$ on the cis side, $250 \mathrm{mM} \mathrm{CsCl}$ on the trans side; 7-10 $\mu \mathrm{M} \mathrm{Ca}^{2+}$; $n=10$ ). Downward deflections represent channel openings. Dashed line to the right of each current trace represents the open state; solid line represents the closed state. Current traces were obtained at a holding potential of $-20 \mathrm{mV}$, filtered at $400 \mathrm{~Hz}$. (B) Current voltage curves showing current amplitudes for WT PC2 and PC2 ${ }^{\text {W414C }}$ obtained at the indicated voltages. Error bars represent the mean \pm SEM. (C) Ciliated cells stably overexpressing Myc epitope-tagged PC2 ${ }^{\text {W414C }}$ (green); cilia marked by anti-acetylated $\alpha$-tubulin (red). There was no PC2 ${ }^{\text {W414C }}$ expression in cilia (arrows). (D) The channel-dead missense variant $\mathrm{PC} 2^{\mathrm{D} 51 \mathrm{~V}}$ with a Myc epitope tag was expressed in cilia (arrows). (E) Truncated Myc epitope-tagged PC2 ${ }^{1703 \mathrm{X}}$ showed strong cilia expression, whereas $(\mathbf{F})$ the chimeric mutant $\mathrm{PC2}{ }^{\text {W414G:L703X }}$, introducing the $\mathrm{PC} 2^{\mathrm{W} 414 \mathrm{C}}$ mutation on the $\mathrm{PC} 2^{\mathrm{L} 703 \mathrm{X}}$ backbone, completely abrogated cilia location despite stable expression of the protein product. The rightmost panel for each series of images in $\mathbf{C}-\mathbf{F}$ represents a merge of the images at left. Scale bars: $5 \mu \mathrm{m}$.

scripts (62). The presence of the dual-epitope tags allowed simultaneous detection of PC1-CTF and PC1-NTF in tissue (Figure 5, A and B). All 4 transgenic lines showed that PC1 exists primarily as a cleaved protein in vivo (Figure 5, A, B, and D). We found that PC1 from the $P k d 1^{F / H}-B A C$ transgene was expressed most strongly in membrane-enriched fractions from lung tissue, while kidney and heart showed moderate expression (Figure 5D). PC1 expression in other tissues was difficult to detect by IB of membrane-enriched fractions (Figure 5D, left panel); expression in these tissues was detectable by IP followed by IB and longer exposures (Figure 5D, right panel). It is also notable that PC1 expression has been reported to be downregulated soon after birth $(63,64)$. Consistent with this, we found that detection of PC1 expression was markedly reduced in mice after 2 weeks of age (data not shown). The patterns of expression we observed were reproduced among several transgenic lines (data not shown), showing that BAC transgenic expression of $\mathrm{PC} 1$ is indeed faithful to the native protein pattern of expression.

The BAC transgenic lines showed that the overall abundance of PC1 in all tissues was very low. Even using antiepitope antibodies required either enrichment of membrane fractions in tissue lysates (Figure 5, A-D) or IP followed by IB (Figure 5D) to reliably detect the protein in native tissues. As a consequence of this low abundance, we were unable to reliably detect PC1 in native tissue immunohistochemistry. We therefore prepared MEFs from $P k d 1^{F / H}-B A C$ transgenic and nontransgenic mice and confirmed expression and GPS cleavage of PC1 in the MEFs (Figure 5E). We were able to detect the epitope-tagged $\mathrm{PC} 1$ in cilia of MEFs, confirming expression of the transgene-encoded protein in cilia (Figure $5 \mathrm{~F}$ and ref. 2). The data support the conclusion that modified BAC transgenic lines are an effective model for studying PC1 in vivo.

Effects of PC1 mutations in native tissues. The dual-epitopetagged $P k d 1^{F / H}-B A C$ transgene can serve as a platform for in vivo structure function studies of mutations in Pkd1. To establish this model, we introduced the PC1 ${ }^{\mathrm{L} 3040 \mathrm{H}}$ GPS mutation into the 

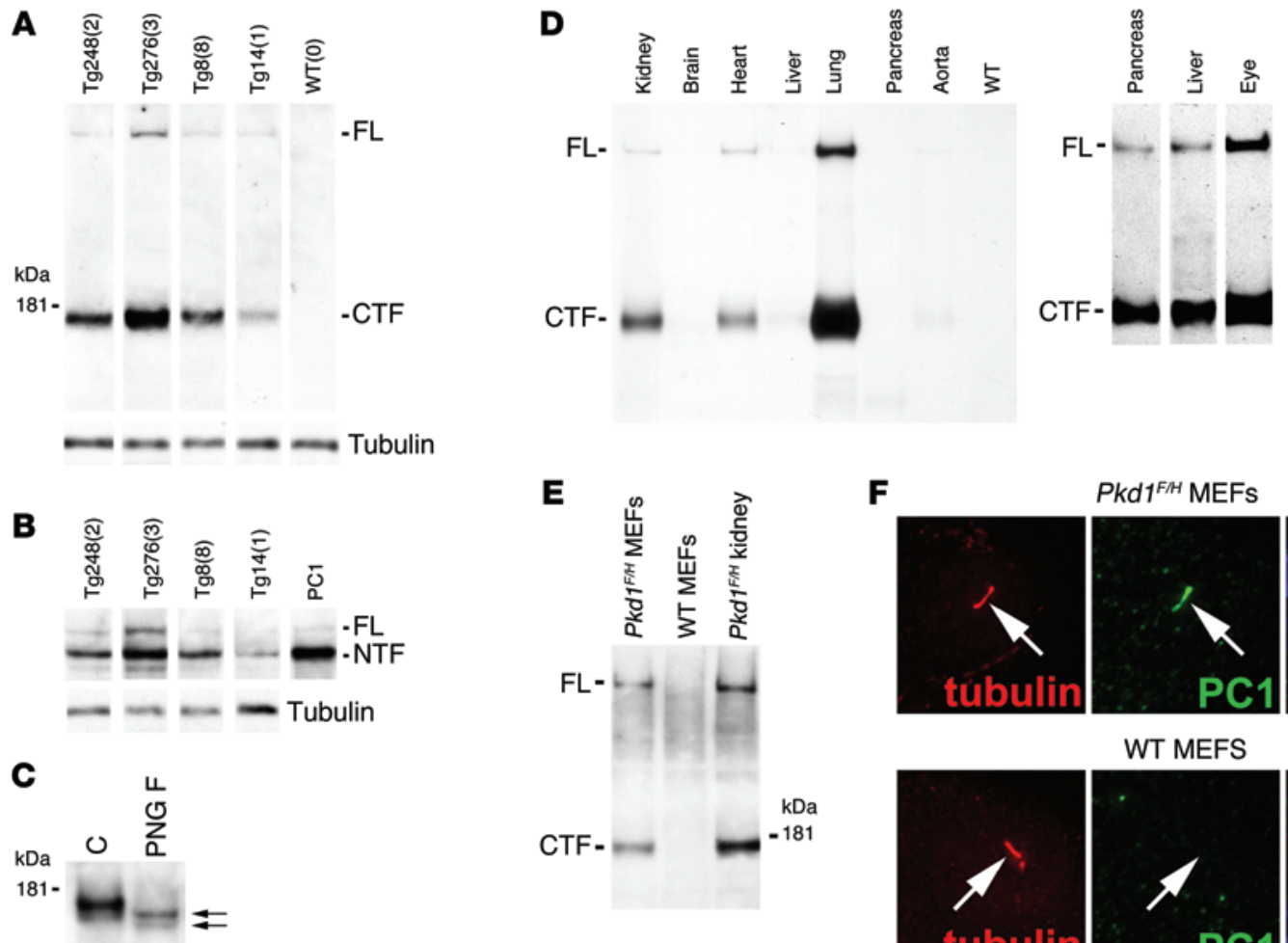

$P k d 1^{F / H}$ MEFs
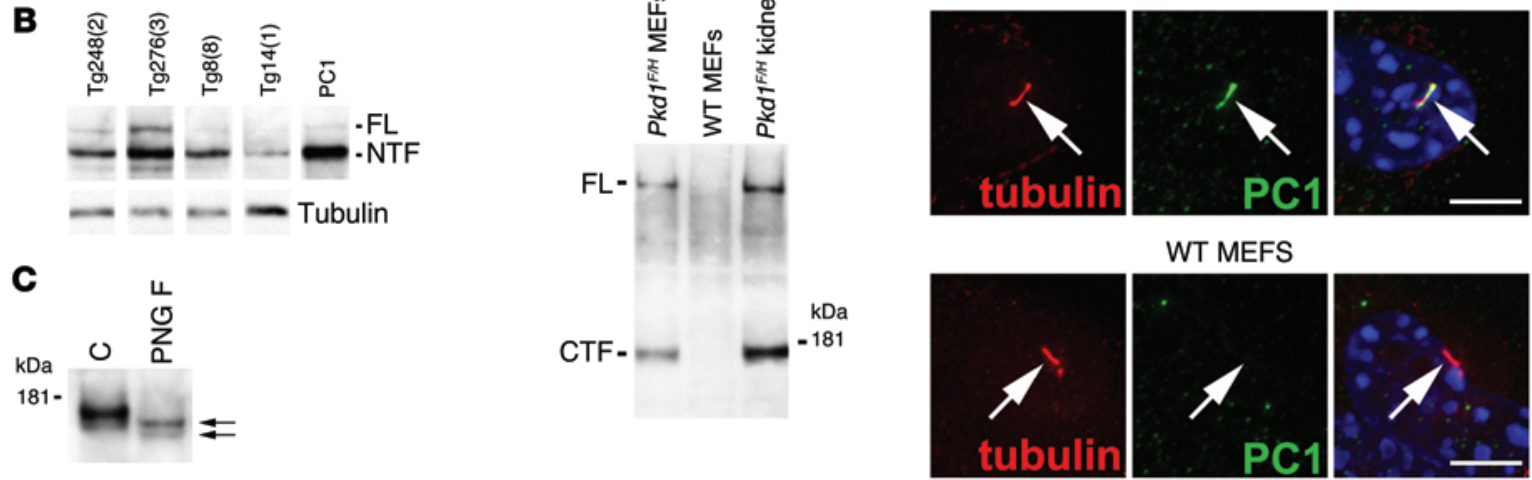

Figure 5. PC1 expression in Pkd1 ${ }^{\mathbf{F} / H}$-BAC transgenic mouse lines. (A and B) Membrane-enriched protein fractions prepared from kidneys of 2-week-old mice descended from 4 independent transgenic founders ( $\operatorname{Tg} 248, \operatorname{Tg} 276, \mathrm{Tg} 8$, and $\mathrm{Tg} 14$; transgene copy number is in parentheses) analyzed by IB with anti-HA (A) and anti-FLAC (B). WT, nontransgenic littermate; PC1, cell lysate overexpressing PC1. Tubulin served as a loading control. Panels represent noncontiguous lanes on a single gel. (C) Evidence of alternative splicing of PC1 expressed from the mouse $P k d f^{F / H}$ - BAC transgene. Kidney tissue lysates from Tg248 underwent IP with anti-HA and were treated with PNGase F or buffer without enzyme (C) prior to IB with anti-HA. Arrows indicate CTF forms with differing migration after PNGase F. (D) Membrane-enriched protein fractions from the indicated organs of a 2-week-old Tg248 mouse underwent IB using anti-HA to show the relative tissue expression of transgenically expressed PC1 (left panel). IP followed by IB of PC1 using anti-HA (right panel) shows detectable levels of $P C 1$ in organs in which IB alone was not sufficiently sensitive. Panels represent noncontiguous lanes on a single gel. (E) $P k d 7^{F / H}-B A C$ kidney tissue ( $P k d 7^{F / H}$ kidney), MEFs ( $P k d 7^{F / H}$ MEFs), and nontransgenic (WT MEFs) cell lysates subjected to IB with anti-HA show expression and GPS cleavage of PC1 in MEFs. (F) PC1 from the Pkd7 $7^{F / H}$-BAC was expressed in cilia of MEFs from Tg248 mice. Green, anti-HA; red, anti-acetylated $\alpha$-tubulin. The rightmost panels in $\mathbf{F}$ represent a merge of the images at left. Scale bars: $5 \mu \mathrm{m}$.

genomic $P k d 1$ sequence in $P k d 1^{F / H}-B A C$ and generated transgenic mice with the modified $P k d 1^{13040 H_{-}}-B A C$ transgene. We obtained 3 transgenic founders with germline transmission: 2 lines ( $T g 46^{L 3040 H}$ and $\mathrm{Tg} 9^{\mathrm{L} 3040 \mathrm{H}}$ ) had 1 copy of the BAC transgene by genomic qPCR, whereas the third line $\left(\mathrm{Tg} 7^{\mathrm{L} 3040 \mathrm{H}}\right)$ had 3 copies (Supplemental Table 5). All 3 transgenic lines showed normal histology at 6 months on a WT background, with no evidence of microscopic cysts in the kidney, liver, or pancreas (data not shown). PC1 expressed from $P k d 1^{L 3040 H}-B A C$ appeared as a single band migrating as PC1-FL without evidence of cleavage at the GPS (Figure 6A). The tissue pattern of expression was similar to that of WT $P k d 1^{F / H}-B A C$ (Figure $6 \mathrm{~B}) . \mathrm{PC}^{\mathrm{L} 3040 \mathrm{H}}$ retained the capacity to interact with $\mathrm{PC} 2$, as evidenced by cell-based cotransfection, co-IP studies (Supplemental Figure 4). None of the $P k d 1^{\mathrm{L3O4OH}}-\mathrm{BAC}$ transgenic lines were able to rescue the embryonic lethality of the $\mathrm{Pkd1}^{-/-}$mice (Figure 6C and Supplemental Table 5), indicating a complete loss-of-function allele based on a single codon change in the entire BAC insert sequence that included $P k d 1$ and the other "passenger" genes. We evaluated the ability of $P k d 1^{L 304 O H}-B A C$ transgenic lines to specifically rescue the cystic phenotype following kidney-selective conditional inactivation of $P k d 1$. $P k d 1^{L 3040 H_{-}}-B A C$ $\left(\mathrm{Tg} 46^{\mathrm{L3O4OH}}\right.$ and $\mathrm{Tg} 7^{\mathrm{L3O4OH}}$ ) did not ameliorate the postnatal kidney cystic phenotype in $P k d 1^{f / f l} \mathrm{Ksp}$-Cre mice, which developed cysts in the medullary thick ascending loop of Henle (mTAL), the distal convoluted tubule (DCT), and the collecting duct (Figure 6D and ref. 65). We found that the same was true in $P k d 1^{f / f l} \mathrm{Pkhd1-Cre}$ mice, in which the cysts were confined to the collecting duct (Figure 6E). IP followed by IB of cystic tissue lysates from both models documented expression of the $\mathrm{PC} \mathrm{L}^{\mathrm{L} 3040 \mathrm{H}}$ protein (Figure 6, F and $\mathrm{G})$. In aggregate, these data indicate complete loss of PC1 function in GPS cleavage-deficient $\mathrm{PC}^{\mathrm{L} 3040 \mathrm{H}}$ in vivo.

Aberrant trafficking and distinct pools of polycystins in native tissues. We took advantage of the epitope-tagged BAC transgenic models to examine the trafficking properties of WT PC1 and the mutant $\mathrm{PC}^{\mathrm{L} 3040 \mathrm{H}}$ in vivo. PC1-CTF from WT $P k d 1^{F / H}-B A C$ transgenic brain tissue showed endo $\mathrm{H}$-resistant and -sensitive forms in vivo (Figure 7A). Uncleaved PC1-FL and $\mathrm{PC1}^{\mathrm{L} 3040 \mathrm{H}}$ were completely sensitive to endo $\mathrm{H}$ (data not shown). For tissue fractionation studies, we used lung tissue, because it showed the most robust expression of both forms of PC1 by IB (Figure 5D and Fig- 

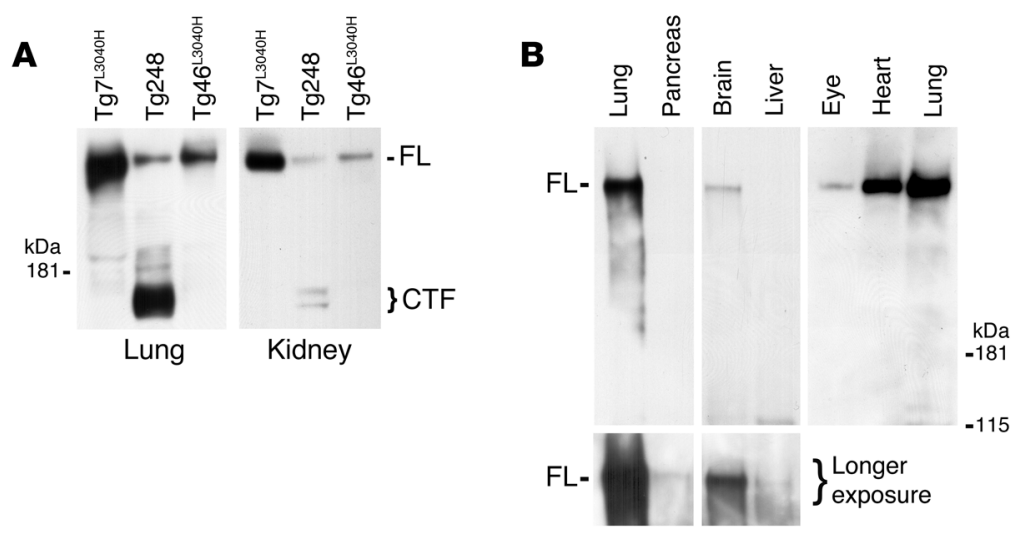

C

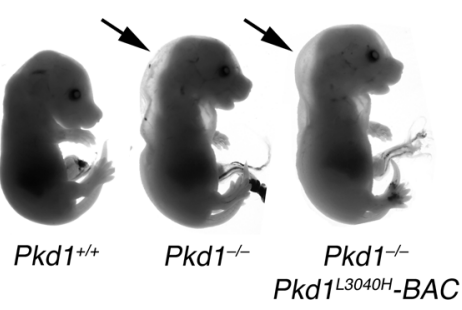

D

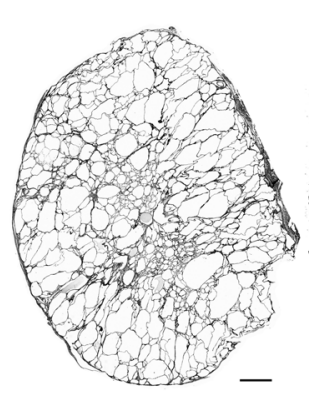

$P k d 1^{t / f t} K s p-C r e$

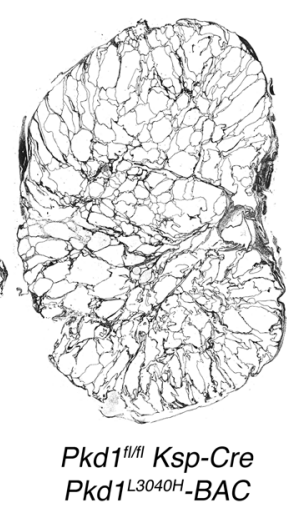

(Tg46 $\left.{ }^{\text {L3040H }}\right)$
E

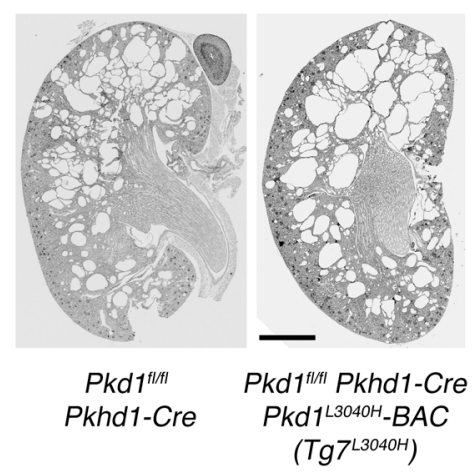

$\mathbf{F}$
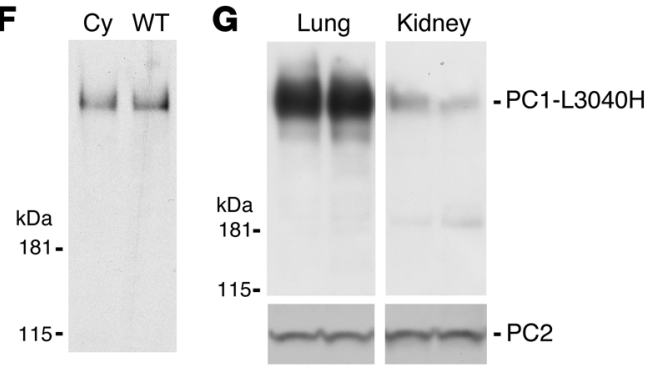

Figure 6. The $\mathrm{PC}^{\mathrm{L}{ }^{2040 \mathrm{H}}}$ mutation results in loss of function in vivo. (A) Immunoblots showing expression of $\mathrm{PC} 1^{\mathrm{L} 3040 \mathrm{H}}$ as uncleaved full-length (FL) protein

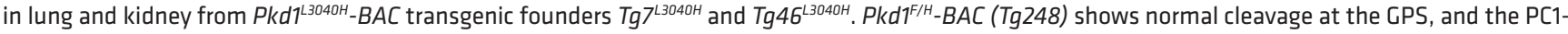
CTF appears as 2 bands due to alternative splicing. Total tissue lysates were subjected to IP and IB with anti-HA. Panels represent noncontiguous lanes from a single gel. (B) Tissue expression of PC1 ${ }^{13040 \mathrm{H}}$ from 2-week-old $P k d 1^{13040 \mathrm{H}}-B A C\left(T g 46^{L 3040 \mathrm{H}}\right)$ transgenic mice. PC1 $1^{\mathrm{L3} 3040 \mathrm{H}}$ was subjected to IP and IB with anti-HA. The 2 left panels represent noncontiguous lanes from a single gel; right panel represents a separate gel. (C) Pkd1 ${ }^{-/-}$embryos at E16.5 had indistinguishable lethal phenotypes including edema (arrows) with or without the $P k d 1^{13040 \mathrm{H}}-B A C$ transgene, indicating that $P C 1^{13040 \mathrm{H}}$ did not reconstitute $P C 1$

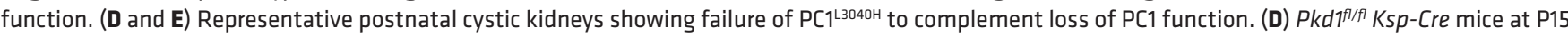

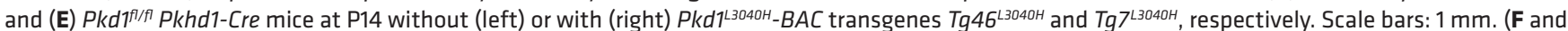

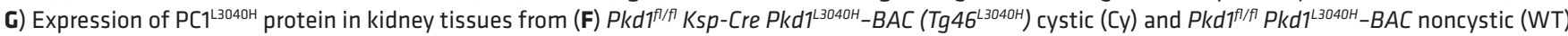
mice at P5 and from (G) a pair of littermate $P k d 1^{f /-} P k h d 1-C r e ~ P k d 1^{23040 H}-B A C\left(T g 7^{L 3040 H}\right)$ polycystic mice at P15 showing that expression of the uncleaved protein was present in the cystic kidney tissues despite a failure to rescue the PKD. PC ${ }^{13040 \mathrm{H}}$ was detected by IP and IB with anti-HA. Panels represent noncontiguous lanes from a single gel.

ure $6 \mathrm{~B})$. We fractionated lung tissue lysates on iodixanol-based linear density gradients and compared the distribution of $\mathrm{PC} 1$ and $\mathrm{PC}^{\mathrm{L} 3040 \mathrm{H}}$ (Figure 7, B and C). We found that WT PC1-NTF and PC1-CTF mainly distributed in the lighter membrane fractions at the top of the gradient that were also enriched in NaK-ATPase, a plasma membrane protein (Figure 7B). Uncleaved PC1-FL, as well as a small amount of PC1-NTF, but not PC1-CTF, appeared in fractions enriched for the ER membrane protein calnexin (Figure 7B). Fractionation of tissue from $\mathrm{PC}^{\mathrm{L}}{ }^{\mathrm{BO} 40 \mathrm{H}}$ transgenic mice showed that the full-length, uncleaved protein was retained entirely in fractions marked by the ER protein calnexin (Figure 7C). PC1 ${ }^{\mathrm{L3} 304 \mathrm{H}}$ was absent from fractions most enriched for NaK-ATPase (Figure $7 C)$. We confirmed that the product detected by anti-FLAG in the $\mathrm{PC}^{\mathrm{L} 3040 \mathrm{H}}$ transgenic line was a full-length protein by running it alongside fractions from the WT PC1 gradient (Figure 7D). The data show in vivo fractionation of functional $\mathrm{PC} 1$ and the disrupted subcellular distribution in tissue of a loss-of-function mutant form, $\mathrm{PC}^{\mathrm{L}}{ }^{\mathrm{L} 040 \mathrm{H}}$. In tissue, full-length, uncleaved WT PC1 or cleavage-deficient mutant $\mathrm{PC}^{\mathrm{L}}{ }^{\mathrm{L} O 40 \mathrm{H}}$ remained in the ER compartment, while the cleaved PC1 products trafficked to the cell surface.

We had previously shown that endogenous PC2 distributes primarily in fractions associated with ER membranes in native kidney tissues (17). We took advantage of the linear-gradient fractionation of tissue from the Pkd1-BAC transgenic mice to examine the relative distributions of $\mathrm{PC} 1$ and $\mathrm{PC} 2$ in tissue (Figure 7, B and C). The fractions in which PC2 was most abundant were largely devoid of PC1-CTF and PC1-NTF; conversely, PC1-CTF and PC1-NTF appeared predominantly in fractions that contained only a minority of tissue PC2 (Figure 7B). The tissue distribution of PC2 did not change with $\mathrm{PC}^{\mathrm{L} 3040 \mathrm{H}}$ expression (Figure $7 \mathrm{C}$ ), consistent with our previous finding that PC2 traffics independently of PC1 (13). The relatively disparate tissue distribution of PC1 and PC2 supports previous data that PC2 functions as an ER channel independently of PC1 $(17,18)$ and that PC2 has functions independent of PC1 $(15$, 

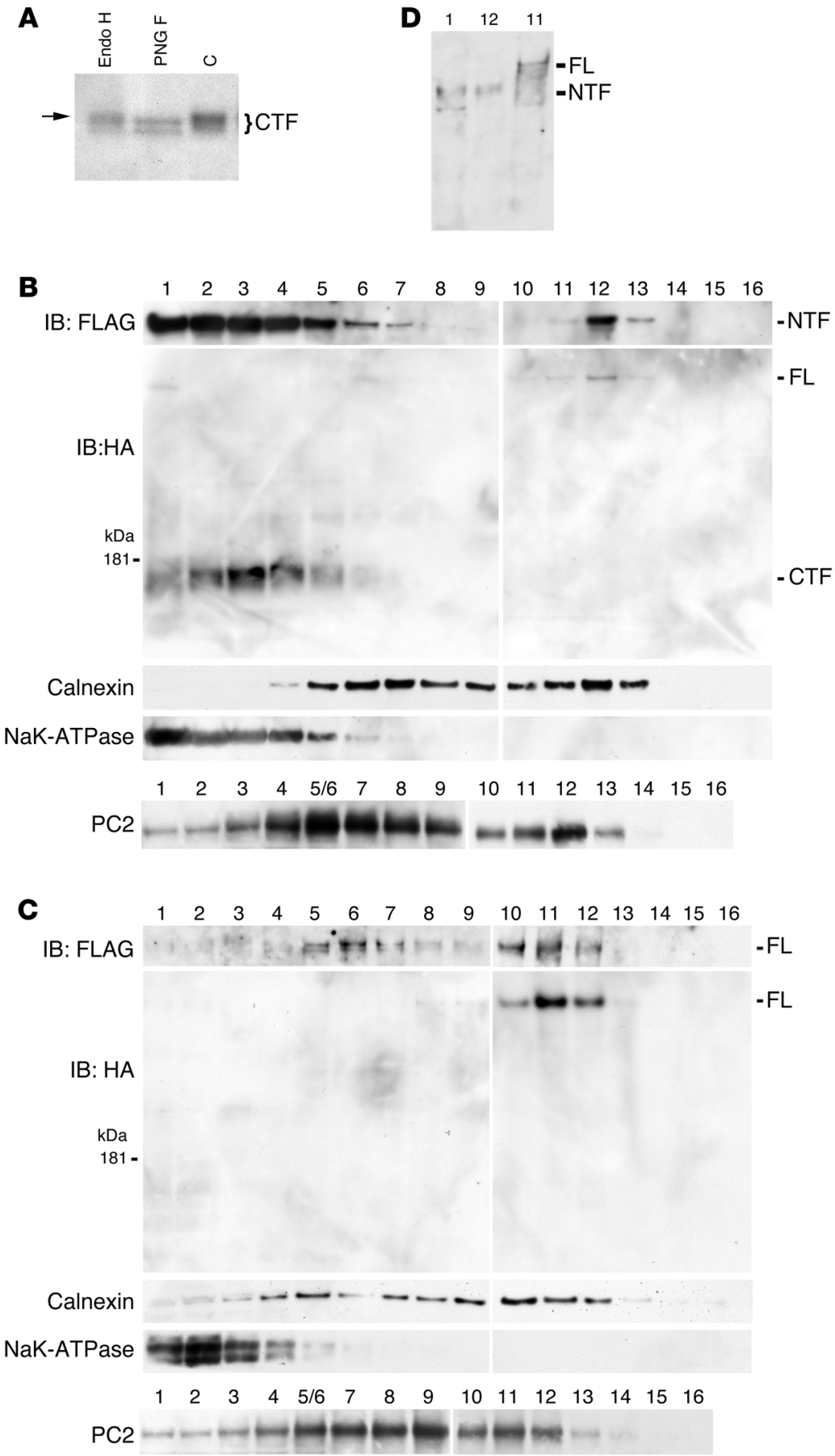

Figure 7. Altered trafficking of PC1 in vivo. (A) Presence of endo $\mathrm{H}$-resistant PC1-CTF in tissues (arrow). PC1 from brain tissue lysates of $P k d 1^{F / H}-B A C(\operatorname{Tg} 8)$ mice was subjected to IP using anti-HA and treated by endo $\mathrm{H}, \mathrm{PNG}$ ase $\mathrm{F}$, or incubated with reaction buffer alone (C), followed by IB with anti-HA. (B and $\mathbf{C}$ ) Distribution of PC1 (B) and PC1 ${ }^{13040 \mathrm{H}}$ (C) in density fractions from tissue lysates. Lung tissue lysates from $P k d 7^{F / H}-B A C(\operatorname{Tg} 8)(B)$ and $P k d 1^{23040 H}-B A C$ $\left(T g 7^{(3040 H}\right)$ (C) mice were fractionated by density-gradient centrifugation on $5 \%$ to $25 \%$ linear iodixanol density gradients. Equal volumes of 16 fractions were loaded in each lane: fraction 1 is the top of the gradient; fraction 16 is the bottom. Samples were analyzed by IB with antibodies against FLAG, HA, calnexin, NaK-ATPase, and PC2. Left and right panels represent separate gels. FLAG, HA, calnexin, and NaK-ATPase were detected on the same gels; PC2 was detected on a separate gel, in which fractions 5 and 6 were combined into a single lane. (D) Fractions 1 and 12 from $\mathbf{B}$ were run alongside fraction 11 from $\mathbf{C}$ and subjected to IB with anti-FLAG to confirm the differential migration of the PC1-NTF bands detected in B and $\mathrm{PC1}-\mathrm{FL}$ detected in $\mathbf{C}$

level overexpression systems using heterologous promoters, we established conditionally immortalized renal epithelial cell lines from mTAL of $P k d 2^{f / f t}$ $P k d 1^{F / H_{-}} B A C$ mice carrying the ImmortoMouse (H-2Kb-tsA58) transgene (Figure 8). These parental cells underwent transient expression of adenovirus-expressed Cre recombinase (adeno-Cre) followed by cloning using limiting dilution. Cells infected with adeno-Cre yielded $P k d 2^{-/-} P k d 1^{F / H_{-}}$ $B A C$ cells that did not express PC2, while cells not infected with adeno-Cre gave $P k d 2^{f / f l} P k d 1^{F / H}-B A C$ cells that retained normal PC2 expression (Figure 8, $A$ and B). Cells grown for 7 to 10 days under nonpermissive conditions exhibited epithelial properties including lateral ZO-1 and E-cadherin staining (Figure 8A and data not shown). Since these cells all carry the same 3-copy $P k d 1^{F / H}-B A C$ transgenic insertion, expression of the epitope-tagged PC1 protein is expected to be constant between cell lines. We found that the steady-state expression levels of PC1-CTF in $\mathrm{Pkd2}^{-/}$cells were markedly reduced compared with those in $P k d 2^{A / f l}$ cells that still express PC2 (Figure $8 \mathrm{C}$ ). This did not reflect a global decrease in PC1 levels, as there was no change in the steady-state

66). The data further suggest that while only a minor proportion of PC2 appears in the same fractions with the PC1-CTF and PC1-NTF, this subfraction may represent the proposed PC1-PC2 functional complex $(3,4,67)$ that is important in the pathogenesis of PKD.

PC1-CTF expression levels are markedly reduced in the absence of PC2. Finally, we sought to examine the unresolved question of the impact of inactivation of $P k d 2$ on PC1 expression. Our observation that overexpression of WT PC2, but not the truncated form $P C 2^{\mathrm{L} 703 \mathrm{X}}$, increased the steady-state levels of PC1-CTF (Supplemental Figure 4) led us to question what effect the absence of PC2, as occurs in cyst cells in patients, has on PC1. To test this directly and to avoid potentially artefactual results due to high- expression of PC1-FL (Figure 8, C and E). This also did not reflect a decreased efficiency of cleavage at the GPS, as we observed no change in PC1-NTF steady-state expression levels between $P k d 2^{f / A l}$ and $P k d 2^{-/}$cells (Figure 8D), nor was there a reciprocal increase in $\mathrm{PC} 1-\mathrm{FL}$ in $\mathrm{Pkd2} 2^{--}$cells (Figure $8, \mathrm{C}$ and E). These results suggest that PC2 is not required for GPS cleavage of PC1. To determine whether the decrease in steady-state levels of PC1-CTF was the result of hyperubiquitination and degradation via the proteasome, we treated $P k d 2^{-/-}$cells with the irreversible proteasome inhibitor carfilzomib (Supplemental Figure 5). As a positive control, HIF1 $\alpha$ levels were stabilized in the presence of carfilzomib. We observed that PC1-CTF did not increase with carfilzomib treatment, indi- 
A

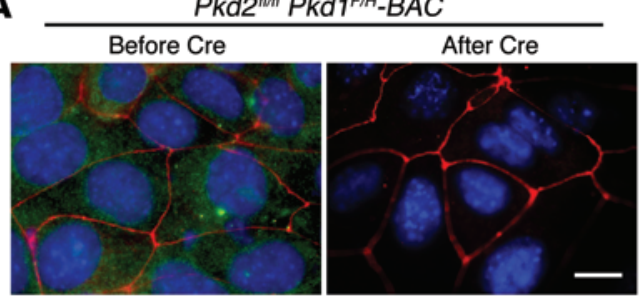

PC2/ZO-1/DAPI

D

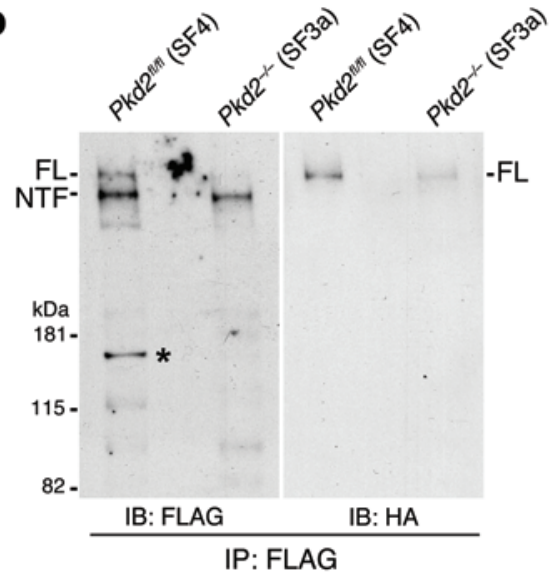

B

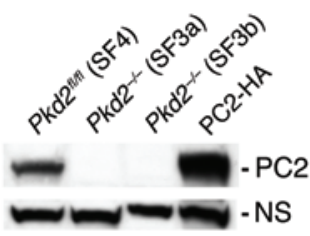

C

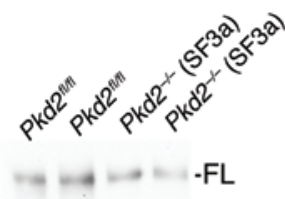

IP: $H A$

$\mathrm{kDa}$

IB: HA

-CTF

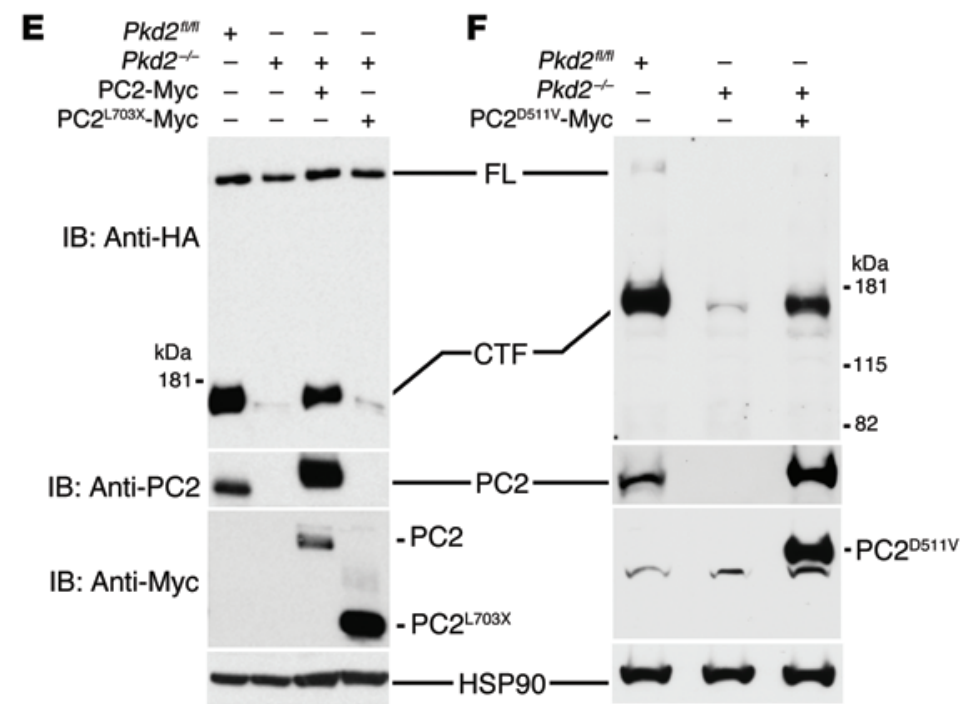

Figure 8. Loss of PC1-CTF expression in the absence of PC2. (A) Conditionally immortalized renal epithelial cell lines from $P k d 2^{f / / f l} P k d 7^{F / H}-B A C(T g 248)$ mice before and after $P k d 2$ inactivation in vitro by transient expression of Cre recombinase. Immunostaining showing loss of PC2 (green) after Cre expression with preserved tight junction formation (ZO-1) in both cell lines. Scale bar: $5 \mu \mathrm{m}$. (B) Immunoblots using anti-PC2 (YCC2) showing PC2 expression in $P k d 2^{f / f l} P k d 1^{F / H}-B A C$ cells and an absence of PC2 in $2 P k d 2^{-1-} P k d 7^{F / H}-B A C$ cell lines. PC2-HA, cell lysate overexpressing PC2. Cell line names: SF4, Pkd2 f//f $P k d 7^{F / H}-B A C ;$ SF3a, SF3b, 2 independent $P k d 2^{-1-} P k d 7^{F / H}-B A C$ cell lines. NS, nonspecific band used as a loading control. (C) Markedly reduced level in PC1-

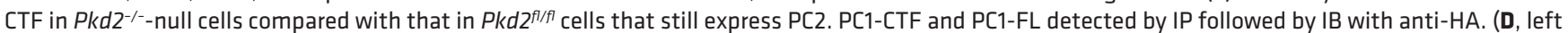

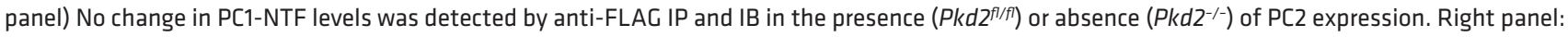
Same gel reprobed with anti-HA to show the relative migration of PC1-NTF. ${ }^{*}$, nonspecific band. (E) Immunoblots of control $P k d 2^{f / / f l} P k d 7^{F / H}-B A C$ cells (left lane) and $P k d 2^{-1-} P k d 7^{F / H}-B A C$ cells reoverexpressing WT PC2-Myc or $\mathrm{COOH}$-terminal truncated PC2 ${ }^{\mathrm{L} 703}$-Myc, showing that only full-length $\mathrm{PC2}$ reconstituted PC1-CTF expression levels. Expression of PC2 had no effect on PC1-FL expression. (F) Reoverexpression of the channel-dead pathogenic PC2 ${ }^{\text {D511v }}$ missense mutant also reconstituted the expression levels of PC1-CTF in $P k d 2^{-1-} P k d 1^{F / H}-B A C$ cells.

cating that increased degradation by the proteasome was not the cause of reduced PC1-CTF in the absence of PC2. The decrease in steady-state levels of PC1-CTF appeared to be a unique effect of PC2 on PC1; loss of PC1 had no effect on the steady-state expression levels of PC2 (Supplemental Figure 6). The findings suggest that PC2, which interacts with PC1-CTF, is significantly required to maintain the steady-state stability of PC1-CTF by a mechanism not dependent on the proteasome.

We used lentivirus-mediated reexpression of WT and mutant forms of $\mathrm{PKD} 2$ in $\mathrm{Pkd}^{-/-}$cells to demonstrate the requirement for interaction between PC2 and PC1 to maintain stable PC1-CTF expression. Re-expression of $\mathrm{PKD} 2$ in $\mathrm{Pkd2^{-- }}$ cells successfully rescued stable PC1-CTF expression (Figure 8E). Lentivirus-mediated reexpression of a truncated form of $\mathrm{PC} 2\left(\mathrm{PC}^{\mathrm{L} 703 \mathrm{X}}\right)$ that lacks the domain for interaction with PC1 failed to rescue PC1-CTF expression (Figure 8E), suggesting that interaction between the 2 polycystin proteins is required for PC1-CTF stability. A channel-dead pathogenic point mutant, $\mathrm{PC} 2^{\mathrm{D} 511 \mathrm{v}}$, which retains the $\mathrm{PC} 1$ interaction domain, was able to fully rescue PC1-CTF expression in $P k d 2^{---}$cells (Figure 8F). These data suggest that PC2 interaction is sufficient for stabilizing PC1-CTF, and this latter function does not require the channel activity of PC2. It is noteworthy that the majority ( $85 \%)$ of PC2 mutations in patients are truncating and expected to abrogate interaction with PC1. These data pose the novel possibility that the pathogenic effect from truncating mutations in PC2 includes a reduced stability of PC1-CTF below a critical threshold and that cyst formation in $P K D 2$ patients results, at least in part, from a reduced functional dosage of PC1, akin to the mechanism for cyst formation in isolated human polycystic liver diseases (2). The pathogenicity of $\mathrm{PC} 2^{\mathrm{D} 511 \mathrm{v}}$ in patients further suggests that the channel activity of PC2 is required for functional integrity of the PC1-PC2 complex independently of PC1-CTF levels in preventing ADPKD.

\section{Discussion}

Recent data have established the role of PC1 dosage as the rate-limiting determinant of ADPKD and ADPLD severity (2, 47, 
68). These findings raise the possibility that targeted personalized therapeutic strategies based on the specific nature of the mutations (69) may be applicable to ADPKD. Success in this approach will require an understanding of the specific functional consequences associated with patient mutations. While the pathogenic principles of protein-destructive chain-terminating mutations in polycystins are understood, less is known about the functional effects of nonsynonymous amino acid substitution mutations. These missense variants may be the most likely initial targets for mutationspecific therapies in ADPKD. Nearly half of the predicted pathogenic mutations associated with human Mendelian disorders involve single amino acid substitutions (70). Loss of normal protein folding or trafficking features prominently among missense variants for which the mechanism is known (70) and plays important roles in diseases such as cystic fibrosis, Alzheimer's disease, Parkinson's disease, and the amyloidoses $(69,71,72)$. Functional defects resulting from missense mutations thought to be pathogenic in ADPKD include loss of GPS cleavage (73) and changes in protein folding and trafficking (47) in PC1 and loss of channel activity in PC2 (17). Our current study used a systematic strategy for categorizing the pathogenic consequences of missense variants in PC1 into: (a) variants that do not undergo GPS cleavage; (b) variants that do not traffic properly to cilia despite undergoing GPS cleavage; and (c) variants that are functionally defective despite normal GPS cleavage and trafficking to cilia. For PC2, missense variants can so far be subcategorized into: (a) variants with loss of channel activity but normal trafficking and (b) variants that retain channel activity but do not traffic normally to cilia.

We developed an in vitro assay for trafficking of PC1-CTF and PC1-NTF as well as PC1-FL and found that PC1 expression in cilia

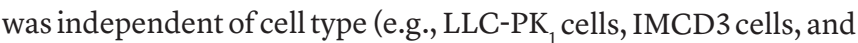
MEFs). PC1-CTF is preferentially biotinylated on the apical surface of live, polarized LLC-PK 1 cells, suggesting that the apical surface and cilia are the primary sites of PC1 surface expression, although there may be some variation by cell type (20). The preponderance of evidence shows that only the PC1-CTF and PC1-NTF cleavage products can traffic to cilia. We found that at least 2 independent GPS cleavage-deficient mutants did not traffic to cilia, and both were complete loss-of-function of mutants: $P C 1^{\mathrm{L} 3040 \mathrm{H}}$ by virtue of its inability to rescue any part of the $P k d 1$-mutant mouse phenotype and $P C 1^{\mathrm{L} 2811 \mathrm{P}}$ by virtue of its pathogenicity in human disease. There is some evidence that cleavage at the GPS may not be an absolute requirement for PC1 to enter the secretory pathway. An engineered mutant $\mathrm{PC} 1, P k d 1^{V / V}\left(\mathrm{PC}^{\mathrm{T} 3041 \mathrm{~V}}\right)$, has been reported to acquire endo $\mathrm{H}$ resistance (50), however, this mutant also behaves as a hypomorphic partial loss-of-function allele that produces a de novo PC1 cleavage product (73), thereby raising the possibility that it is not entirely reflective of GPS cleavage-defective mutants in general. Additionally, we showed that at least 2 pathogenic variants, $P C 1^{\mathrm{C} 210 \mathrm{G}}$ and $P C 1^{\mathrm{V} 685 \mathrm{D}}$, which do not affect GPS cleavage, also failed to localize to cilia. In aggregate, the data suggest that pathogenic point mutations affecting conformational changes, with or without impairing GPS cleavage, can nonetheless impair cilia trafficking. In the current study, 4 of the 5 PC1 missense mutations we examined failed to traffic to cilia, which poses the hypothesis that a majority of pathogenic point mutations in PC1 have failure to traffic to cilia as a common biologic feature.
Aside from conformational changes, we also found that part of the $\mathrm{COOH}$ terminus region of PC1 was necessary for trafficking out of the ER and into cilia. The pathogenic truncation mutant $\mathrm{PC}^{\mathrm{R} 4204 \mathrm{X}}$ entered cilia, indicating that the coiled-coil domain (residues 4,214-4,248) thought to mediate interaction with PC2 (53, 74 ) is not required for trafficking PC1 to cilia. Complete deletion of the $\mathrm{COOH}$ terminus in $\mathrm{PC}^{\mathrm{Y} 4100}$ shows a novel functional requirement of the region between $\mathrm{PC} 1^{\mathrm{Y} 4100}$ and $\mathrm{PC}^{\mathrm{R} 4204}$ for PC1 trafficking. While it is possible that this region is required to maintain conformational integrity to allow trafficking much the way point mutations do, it is more likely that this region of the $\mathrm{COOH}$ terminus is required for interaction with factors that are critical for PC1 egress from the ER. Not all point mutations in PC1 affect cilia trafficking. A mutation in a conserved residue in one PKD domain, $\mathrm{PC}^{\mathrm{G} 1160 \mathrm{~s}}$, remains competent in GPS cleavage, cilia trafficking, and interaction with PC2, but still lacks normal function. Mutations in PKD domains may be part of a class of variants deficient in PC1 functions, perhaps related to ligand interaction, which are required once the protein is in cilia. Experimentally, this type of mutation may serve as an appropriate entry point for discovery of the cilia-specific functions of $\operatorname{PC} 1(75,76)-$ i.e., if a cellular or physiological response is attributed to PC1 specifically in cilia, then expression of these mutants in place of WT protein would be expected to result in loss of the specific response.

While amino acid substitution variants are less common in PC2 and therefore less likely to be targets for therapy, they can nonetheless be instructive in discerning function for the polycystins. The pathogenic missense variant $\mathrm{PC}^{\mathrm{D} 511 \mathrm{v}}$ has complete loss of channel activity (17) but traffics to cilia, whereas a variant in a highly conserved residue, $\mathrm{PC} 2^{\mathrm{W} 414 \mathrm{G}}$, which has largely preserved channel function, does not traffic to cilia. These data provide support for the hypothesis that both cilia location and channel activity in cilia are required for polycystin function $(15,16)$. On the other hand, recent data directly examining ciliary calcium suggest that calcium concentrations in cilia are significantly higher than those in the cell body $(75,76)$. To put all of these findings together, the hypothesized role of ciliary polycystins in global cellular calcium responses may require significant revision. The aggregate information available suggests a hypothesis that the ciliary calcium channel function of polycystins has very local effects, possibly acting only within the polycystin protein complex. Nonetheless, our findings offer the first opportunity to categorize groups of missense mutations in polycystins into functionally distinct subtypes. The groups of variants for both polycystins that do not traffic to cilia may be appropriate to target with therapies aimed at countering protein-folding defects (77) and raise the prospect that such therapies may be applicable to selected patients with ADPKD.

ADPKD is a disease of $3 \mathrm{D}$ organ structure manifest in the complex cellular milieu of the adult kidney. As a consequence, the relevance of mechanistic discoveries based on in vitro cell culture models requires validation with in vivo models. To this end, we developed BAC-recombineering transgenic models for Pkd1 (2). BAC transgenic models have practical advantages over conventional knockin models, including the ability to target the same allele multiple times, the speed with which mutant mouse lines can be obtained, and the reduced cost in doing so. The emergence of genome-editing technologies, specifically the CRSPR/ 
Cas9 system, may offer similar advantages for future studies. Previous reports of Pkd1-BAC transgenic models showed cystic phenotypes that were the result of high transgene copy numbers $(60,78)$ and the use of heterologous promoters (61). Our WT $P k d 1^{F / H}-B A C$ founders were low copy numbers and achieved complete functional rescue of the $P k d 1^{-/-}$phenotype. The mutant $P k d 1^{13040 H}-B A C$ model was true to the in vitro data, producing uncleaved PC1 retained in the ER and resulting in a complete loss-of-function allele in vivo. The finding that the entire functionality of the $B A C$ transgene in multiple founders could be eliminated by a single codon change in only 1 gene, $P k d 1$, eliminated any possibility that the other genes contained in the BAC had any contribution to the observed phenotypic effects in vivo. Our result with a GPS mutant is somewhat different from that of a knockin mouse model with a mutation in an adjacent codon, $P k d 1^{T 3041 V}$ $\left(P k d 1^{V / v}\right)$, which had postnatal survival up to 2 to 6 weeks with polycystic kidneys (73). These phenotypic differences highlight the fact that the specific nature of the genomic alteration and associated amino acid substitution is likely to be a key determinant of the degree of functional inactivation of polycystins.

These in vivo models also allowed us to compare the fractionated tissue distribution of PC1 and PC2. While both proteins are thought to function together in cilia in preventing cyst formation in the kidney, there is ample evidence that they also function independently in various tissues and subcellular locations. PC2 has a role in left-right axis determination, whereas PC1 does not (14, 66). PC2 is abundantly expressed in the ER, where it has channel activity, but that role appears to be independent of PC1 $(17,18)$. Our WT Pkd1 transgenic lines provided direct evidence that PC1 and PC2 distributed in distinct but overlapping density fractions. The majority of cleaved WT PC1 resides in presumed plasma membrane-associated fractions that contained a minute fraction of total tissue PC2. The existence of the bulk of PC2 protein in ER-associated tissue fractions devoid of cleaved PC1 lends strong support to the hypothesis that PC2 has cellular functions in the ER that are independent of PC1. Whether PC2 function independent of $\mathrm{PC} 1$ has a role in ADPKD remains to be determined.

Finally, we took advantage of our in vivo and in vitro models to examine the interrelationship between PC1 and PC2 expression. Surprisingly, we found that $\mathrm{PC1}-\mathrm{CTF}$ required interaction with $\mathrm{PC} 2$ to maintain steady-state expression levels. The loss of PC1-CTF in the absence of PC2 was not the result of proteasomal degradation. Although not experimentally verified, there have been reports of a PEST domain in the $\mathrm{COOH}$ terminus of PC1 (4), which raises the possibility that the reduced steady-state levels of PC1-CTF in the absence of PC2 are the result of increased degradation by calpain protease (79). In this regard, it is notable that PC1 truncation mutants, in which we eliminated the possible PEST domain and the coiled-coil PC2 interaction domain, nonetheless had sufficient stability to traffic to cilia. Interpretation of these findings must be tempered, however, by the fact that these cells highly overexpressed $P k d 1$ cDNA from a heterologous promoter and cannot be directly compared with a 3-copy $B A C$ transgene in the $P k d 2^{-/-}$cells. A recent study has suggested that PC1-NTF can exist stably without PC1-CTF, but PC1-CTF is critically required for PC1-NTF trafficking (50). In keeping with this, we did not find a profound reduction in PC1-NTF steady-state levels in the absence of PC2. Our data suggest the possibility that one of the pathogenic effects of truncation mutations in PC2 is a markedly decreased steady-state level of PC1-CTF protein, which in turn is critically required for PC1 function. The finding that the channel-dead pathogenic variant $\mathrm{PC} 2^{\mathrm{D} 511 \mathrm{~V}}$ (17) that traffics to cilia in LLC-PK ${ }_{1}$ cells (80) and can rescue the stability of PC1-CTF nonetheless results in human ADPKD (56) suggests that both PC2 interaction with PC1 and PC2 channel activity are independently required for preventing ADPKD.

In aggregate, these studies highlight 5 important findings in the pathogenesis of ADPKD: (a) a subset of pathogenic nonsynonymous substitution mutations results in impaired trafficking of PC1 and PC2 to cilia; (b) mutations that impair GPS cleavage also impair cilia trafficking; (c) cilia-trafficking studies can serve as in vitro assays for PC1 (and PC2) function; (d) the bulk of PC2 resides in the ER without associated $\mathrm{PC} 1$ in vivo; (e) truncation mutations in PC2 result in markedly reduced PC1-CTF protein steady-state expression levels. We propose that cilia-trafficking mutant forms of PC1 may be amenable to therapies directed at assisting protein folding with a readout of successful localization to cilia in vitro as the first step to identifying candidate therapeutic agents. In addition, understanding the molecular basis for the stabilization of PC1-CTF by PC2 interaction could lead to the discovery of agents that increase steady-state levels of PC1 as a therapy for hypomorphic PC1 variants.

\section{Methods}

cDNA constructs, antibodies, and cell culture. The full-length cDNA of mouse $P k d 1$ was cloned, sequenced, verified, and modified with $\mathrm{NH}_{2}$-terminal triple-FLAG and $\mathrm{COOH}$-terminal triple-HA epitope tags as described previously (49). Mutant $P k d 1$ cDNA constructs were generated on this backbone by site-directed mutagenesis using the QuikChange kit (Stratagene). All mutations were confirmed by direct sequencing. LLC-PK 1 cells stably expressing WT and mutant Pkd1 constructs were established as described previously (51). Briefly, cells were transfected with plasmid DNA using Lipofectamine 2000 (Invitrogen) and split at a 1:10 dilution after 24 to 48 hours. Selection with G418 (400-600 $\mu \mathrm{g} / \mathrm{ml})$ was begun after overnight culture. Selected cell clones were characterized by IF cell staining and IB of 50 to $100 \mu \mathrm{g}$ of protein per sample using anti-HA and anti-FLAG antibodies.

Rabbit polyclonal antisera for $\mathrm{PC} 2 \mathrm{COOH}$ terminus (YCC2) and $\mathrm{NH}_{2}$ terminus (YCB9) have been described previously (51). Other antibodies used were from the following sources: mouse monoclonal antiMyc (clone 9E10, 1667149; Roche); rabbit polyclonal anti-Myc (2272; Cell Signaling Technology); anti-c-Myc-conjugated agarose (A7470; Sigma-Aldrich); rat monoclonal anti-HA (clone 3F10, 1867423; Roche); rabbit polyclonal anti-HA (715500; Zymed); mouse monoclonal anti-FLAG M2-conjugated agarose (A2220; Sigma-Aldrich); rabbit polyclonal anti-FLAG (F7425; Sigma-Aldrich); mouse monoclonal anti-acetylated $\alpha$-tubulin (T6793; Sigma-Aldrich); mouse monoclonal anti- $\gamma$-tubulin (T6557; Sigma-Aldrich); mouse monoclonal anti$\beta$-tubulin Cy3 conjugate (C4585; Sigma-Aldrich); mouse monoclonal anti-HA FITC conjugate (H7411; Sigma-Aldrich); mouse monoclonal $\alpha$-subunit of NaK-ATPase clone 6H (gift of Michael Caplan, Yale University, New Haven, Connecticut, USA); and rabbit polyclonal anticalnexin (SPA-860; Stressgen).

Immunocytochemistry, IP, and IB. IF cell staining was performed as described previously $(49,51)$. Briefly, cells grown on coverslips or 
semipermeable filter inserts were fixed with $2.5 \%$ paraformaldehyde and permeabilized with $0.3 \%$ Triton $\mathrm{X}-100$ and $0.1 \%$ BSA in PBS. Blocking was carried out using 3\% BSA, and primary and secondary antibodies were applied at empirically determined dilutions. Cells were examined either by wide-field fluorescence (TE2000U; Nikon) with MetaMorph acquisition software or by confocal microscopy (LSM 510; Zeiss). IP experiments were performed as described previously, with minor modifications $(49,51)$. Cells were lysed with glycerol lysis buffer (12), and lysates were precleared by incubation with protein A for 30 minutes, followed by IP starting with 500 to $1,000 \mu \mathrm{g}$ of protein using anti-HA agarose beads (Sigma-Aldrich) at $4^{\circ} \mathrm{C}$ overnight. The beads were washed with lysis buffer 5 times, followed by SDS-PAGE on $3 \%$ - to $8 \%$-gradient gels and IB as described previously $(49,51)$.

Glycosylation analysis and cell-surface biotinylation. Glycosylation analysis was performed as described previously $(49,51)$. PC1 was was subjected to IP using either FLAG or HA antibodies and incubated with either endo H, PNGase F, or reaction buffer alone (New England Biolabs) at $37^{\circ} \mathrm{C}$ for 1 hour followed by IB. Biotinylation of cell-surface proteins was performed using EZ-Link NHS-SS-Biotin (Pierce Biotechnology) as described previously $(49,51)$. $N$-hydroxysuccinimide-SS-biotin was applied to living cultured cells stably expressing PC1 for 30 minutes at $4^{\circ} \mathrm{C}$. Biotinylated proteins were recovered using streptavidin agarose beads and detected by IB.

BAC modification. The mouse BAC clone RPCI22-287A3 containing $P k d 1$ was modified by homologous recombination in $E$. coli using the method of Lalioti and Heath $(57,58)$ in the Mouse Genetics and Cell Line Core (George M. O'Brien Kidney Center, Yale School of Medicine, New Haven, Connecticut, USA). $P k d 1^{F / H}-B A C$ and $P k d 1^{L 3040 H}-B A C$ were generated by sequential BAC modifications to introduce 3 copies each of HA and FLAG epitopes and then the Pkd1$\mathrm{L} 304 \mathrm{OH}$ mutation. In general, for each modification step, approximately 1.0- to 1.3-kb segments of genomic DNA spanning the sites for the desired variants were amplified by PCR, subcloned into Zero Blunt TOPO (Invitrogen), and sequenced in both directions. In-frame triple$\mathrm{HA}$ and triple-FLAG sequences and the variant encoding the $\mathrm{L} 304 \mathrm{OH}$ mutation were introduced into the respective targeting vector subclones and confirmed by sequencing. These targeting constructs were cloned into the shuttle vector PKO4F-Kan $(57,58)$ containing kanamycin resistance for positive selection and 2 negative selection cassettes, temperature-sensitive RepA-ts (permissive at $30^{\circ} \mathrm{C}$, nonpermissive at $43^{\circ} \mathrm{C}$ ), and $s a c \mathrm{~B}$, which blocks growth on sucrose. DH1OB bacteria containing the parental $\mathrm{BAC}$ clone were made competent by $\mathrm{CaCl}_{2}$ and transformed with pK04F-Kan containing the targeting sequence and the recA vector pDF25-Tet containing tetracycline resistance. The BAC carries chloramphenicol resistance $(\mathrm{cm})$. Cotransformants were selected by kanamycin (pK04F), tetracycline (pDF25-Tet), and $\mathrm{cm}(\mathrm{BAC})$ at $30^{\circ} \mathrm{C}$. Cointegrants were selected by growth at $43^{\circ} \mathrm{C}$ in the presence of kanamycin to select against nonintegrated $\mathrm{pKO} 4 \mathrm{~F}$ shuttle vector and confirmed by sequencing. Confirmed cointegrants underwent resolution (removal of the reduplicated region) by a second round of transformation with the pDF25-Tet recA plasmid, followed by selection with tetracycline and chloramphenicol at $30^{\circ} \mathrm{C}$ overnight and a second round of selection by growth on $5 \%$ sucrose with $\mathrm{cm}$ at $43^{\circ} \mathrm{C}$. Single colonies from this final selection step were grown in parallel in $\mathrm{cm} / \mathrm{Kan} / 5 \%$ sucrose and $\mathrm{cm} / 5 \%$ sucrose only. Resolved clones show growth only on $\mathrm{cm} / 5 \%$ sucrose. Successfully targeted BAC clones were confirmed by sequencing.
Generation and characterization of BAC transgenic mice. DNA from $P k d 1^{F / H}-B A C$ and $P k d 1^{L 3040 H_{-}}-B A C$ mice was prepared using the Maxi Prep Kit (QIAGEN). The BAC was linearized with PI-Sce1, and the digestion product was resolved by field-inversion gel electrophoresis (FIGE) (Bio-Rad). The migration of the high-molecular-weight BAC DNA was inferred by a parallel lane stained with ethidium bromide, the band was excised, and the BAC insert DNA was liberated by digestion with $\beta$-agarase I. The eluate was purified using an Ultrafree-MC filter column (Millipore) to obtain the linearized BAC DNA ready for pronuclear microinjection at a concentration of 2 to $3 \mathrm{ng} / \mu \mathrm{l}$. Potential founder animals were screened for the presence of the $B A C$ transgene by PCR primers. Primers directed to the FLAG and HA regions were also used to eliminate founders with only partially integrated BACs. Genotyping of $P k d 1^{L 3040 H}-B A C$ included primers flanking the GPS followed by BtsI restriction digestion; the site is lost in the mutant allele. The BAC transgenic lines were produced in (C57BL/6 J $\times$ SJL/J) F2 mice, and germline transmission was achieved by mating with C57BL6 mice. All strains were subsequently backcrossed at least 4 generations with C57BL6 mice and are therefore expected to be at least 90\% C57BL6 congenic.

Quantitation of BAC transgene copy numbers. We have adapted a method for assessing $B A C$ transgene copy numbers using TaqManbased real-time PCR (81). TaqMan primers were designed to specifically amplify either the endogenous WT allele or the transgene-specific allele. Genomic DNA was prepared from mouse livers by phenolchloroform extraction. All samples were run in triplicate on the ABI Prism 7300 System (Applied Biosystems). The 2 primer sets showed highly efficient amplification ( $\mathrm{R}=0.992$ and 0.996, respectively), enabling quantitation of $B A C$ transgene copy numbers.

Planar lipid bilayer measurements. Membrane vesicles enriched in THE ER from LLC-PK 1 porcine kidney cell lines stably expressing WT PC2 or containing the $\mathrm{PC} 2^{\mathrm{W} 414 \mathrm{G}}$ mutation were isolated by differential centrifugation in the presence of protease inhibitors as previously described (17). These ER vesicles were fused to lipid bilayers containing phosphatidylethanolamine and phosphatidylserine (3:1 w/w; Avanti Polar Lipids) dissolved in decane (40 mg lipid/ml). In experiments using $\mathrm{Cs}^{+}$as the primary current carrier, ER membranes containing PC2 were added to the cis side, which contained $500 \mathrm{mM}$ $\mathrm{CsCl}, 7 \mu \mathrm{M} \mathrm{CaCl}_{2}, 10 \mathrm{mM}$ HEPES, and $0.2 \mathrm{~mm}$ EGTA, pH 7.35. The trans side, contained $250 \mathrm{mM} \mathrm{CsCl}$ and $10 \mathrm{~mm}$ HEPES, pH 7.35. After fusion of vesicles, the cis side was rapidly perfused with $15 \mathrm{ml}$ of the trans buffer with no added calcium. In all experiments, single-channel currents were measured at various holding potentials with respect to the trans (ground) side.

Tissue fractionation. Lung tissues from $P k d 1^{\mathrm{F} / \mathrm{H}_{-}} \mathrm{BAC}$ and $P k d 1^{\mathrm{L304OH}}$ $B A C$ transgenic mice were fractionated on linear iodixanol OptiPrep (Sigma-Aldrich) gradients as described previously (17), with minor modifications. Briefly, lung tissue homogenates were prepared using the OptiPrep buffer (Axis-Shield PoC AS) and loaded onto a freshly made $5 \%-25 \%$ gradient. Samples were centrifuged at $100,000 \mathrm{~g}$ in a SW28 rotor for 2 hours. Sixteen $\sim 2$-ml fractions were collected, and aliquots of each fraction were analyzed by SDS-PAGE followed by IB.

Generation of kidney cell lines from mutant mice. Kidneys were obtained from $P k d 2^{f / A l} P k d 1^{F / H_{-}}-B A C$ (Tg248) mice carrying the ImmortoMouse IFN- $\gamma$-inducible $\mathrm{H}-2 \mathrm{~Kb}$-tsA58 SV40 temperaturesensitive transgene. Kidney tubule epithelial cell lines were produced as described previously $(2,82)$. Resultant $P k d 2^{f / A l} P k d 1^{F / H}-B A C$ "paren- 
tal" cell lines were converted to $P k d 2^{-/-} P k d 1^{F / H}-B A C$-null cell lines ex vivo by infection with 1 to $10 \mathrm{PFU} / \mathrm{cell}$ of adeno-Cre (0100062001; Eton Bioscience), followed by cloning using limiting dilution. Uninfected cells were used as controls. The $P k d 2^{-/-} P k d 1^{F / H}-B A C$ cell lines used were SF3a and SF3b; the $P k d 2^{f / f} P k d 1^{F / H}-B A C$ cell line used was $\mathrm{SF} 4$. Prior to the experiments, cells were allowed to differentiate following silencing of the SV40 large $\mathrm{T}$ antigen under nonpermissive conditions $\left(37^{\circ} \mathrm{C}\right.$, without IFN- $\gamma$ ) for 7 to 21 days.

Reoverexpression of WT or mutant PKD2 carrying the Myc tag into the $P k d 2^{-/-} P k d 1^{F / H}-B A C$ cells. Target gene cDNA was cloned into the pLVX-IRES-puro lentiviral expression vector (Clontech), followed by cotransfection with the packaging system (pMDLg/pRRE plus pRSVRev plus pMD2.G; Addgene) into 293T cells using the calcium phosphate transfection method. After incubation of the cells in a $37^{\circ} \mathrm{C} \mathrm{CO}_{2}$ incubator for 40 to 48 hours, the culture medium supernatant was collected, filtered, and concentrated by centrifugation. The viruses were resuspended in culture medium and used to infect the $P k d 2^{-/-} P k d 1^{F / H_{-}}$ $B A C$ cells. The infected cells were further selected for 3 to 5 days by puromycin selection at 1 to $4 \mu \mathrm{g} / \mathrm{ml}$. Target gene expression was verified by IB and IF cell staining.
Statistics. Statistical significance was determined by 2-tailed Student's $t$ test, with a $P$ value of less than 0.05 considered statistically significant.

Study approval. All animal studies were reviewed and approved by the IACUC of Yale University.

\section{Acknowledgments}

We thank Peter Igarashi and the University of Texas Southwestern O'Brien Kidney Center for providing the Ksp-Cre and Pkhd1-Cre mouse strains. This work was supported by grants DK54053, DK51041, and DK57328 from the National Institute of Diabetes and Digestive and Kidney Diseases (NIDDK), NIH (to S. Somlo); grant 96a2r from the PKD Foundation (to Y. Cai); and by the Yale O'Brien Center core services (P30 DK079310) and the PKD Core Center (P30 DK090744).

Address correspondence to: Stefan Somlo, Section of Nephrology, Yale University School of Medicine, P.O. Box 208029, 333 Cedar Street, New Haven, Connecticut 06520-8029, USA. Phone: 203.737.2974; E-mail: stefan.somlo@yale.edu.
1. Torres VE, Harris PC, Pirson Y. Autosomal dominant polycystic kidney disease. Lancet. 2007;369(9569):1287-1301.

2. Fedeles SV, et al. A genetic interaction network of five genes for human polycystic kidney and liver diseases defines polycystin- 1 as the central determinant of cyst formation. Nat Genet. 2011;43(7):639-647.

3. Qian F, Germino FJ, Cai Y, Zhang X, Somlo S, Germino GG. PKD1 interacts with PKD2 through a probable coiled-coil domain. Nat Genet. 1997;16(2):179-183.

4. Tsiokas L, Kim E, Arnould T, Sukhatme VP, Walz G. Homo- and heterodimeric interactions between the gene products of PKD1 and PKD2. Proc Natl Acad Sci U S A. 1997;94(13):6965-6970.

5. Barr M, DeModena J, Braun D, Nguyen C, Hall D, Sternberg P. The caenorhabditis elegans autosomal dominant polycystic kidney disease gene homologs lov-1 and pkd-2 act in the same pathway. Curr Biol. 2001;11(17):1341-1346.

6. Lu W, et al. Perinatal lethality with kidney and pancreas defects in mice with a targetted Pkd1 mutation. Nat Genet. 1997;17(2):179-181.

7. Wu G, et al. Cardiac defects and renal failure in mice with targeted mutations in Pkd2. Nat Genet. 2000;24(1):75-78.

8. Praetorius HA, Spring KR. The renal cell primary cilium functions as a flow sensor. Curr Opin Nephrol Hypertens. 2003;12(5):517-520.

9. Nauli SM, et al. Polycystins 12 mediate mechanosensation in the primary cilium of kidney cells. Nat Genet. 2003;33(2):129-137.

10. Huang AL, et al. The cells and logic for mammalian sour taste detection. Nature. 2006;442(7105):934-938.

11. Ishimaru $Y$, Inada $H$, Kubota $M$, Zhuang H, Tominaga $M$, Matsunami $H$. Transient receptor potential family members PKD1L3 and PKD2L1 form a candidate sour taste receptor. Proc Natl Acad Sci US A. 2006;103(33):12569-12574.

12. Hanaoka K, et al. Co-assembly of polycystin-1 and - 2 produces unique cation-permeable currents. Nature. 2000;408(6815):990-994.

13. Geng L, et al. Polycystin-2 traffics to cilia independently of polycystin-1 by using an $\mathrm{N}$-terminal RVxP motif. J Cell Sci. 2006;119(pt 7):1383-1395.

14. Pennekamp P, et al. The ion channel polycystin-2 is required for left-right axis determination in mice. Curr Biol. 2002;12(11):938-943.

15. Yoshiba S, et al. Cilia at the node of mouse embryos sense fluid flow for left-right determination via Pkd2. Science. 2012;338(6104):226-231.

16. McGrath J, Somlo S, Makova S, Tian X, Brueckner M. Two populations of node monocilia initiate left-right asymmetry in the mouse. Cell. 2003;114(1):61-73.

17. Koulen P, et al. Polycystin-2 is an intracellular calcium release channel. Nat Cell Biol. 2002;4(3):191-197.

18. Geng L, et al. Syntaxin 5 regulates the endoplasmic reticulum channel-release properties of polycystin-2. Proc Natl Acad Sci U S A. 2008;105(41):15920-15925.

19. Ibraghimov-Beskrovnaya O, et al. Polycystin: in vitro synthesis, in vivo tissue expression, and subcellular localization identifies a large membrane-associated protein. Proc Natl Acad Sci U S A. 1997;94(12):6397-6402.

20. Boletta A, et al. Biochemical characterization of bona fide polycystin-1 in vitro and in vivo. Am J Kidney Dis. 2001;38(6):1421-1429.

21. Streets AJ, Newby LJ, O'Hare MJ, Bukanov NO, Ibraghimov-Beskrovnaya O, Ong AC. Functional analysis of PKD1 transgenic lines reveals a direct role for polycystin-1 in mediating cell-cell adhesion. J Am Soc Nephrol. 2003;14(7):1804-1815.

22. Roitbak T, Ward CJ, Harris PC, Bacallao R, Ness SA, Wandinger-Ness A. A polycystin-1 multiprotein complex is disrupted in polycystic kidney disease cells. Mol Biol Cell. 2004;15(3):1334-1346.

23. Scheffers MS, et al. Polycystin-1, the product of the polycystic kidney disease 1 gene, co-localizes with desmosomes in MDCK cells. Hum Mol
Genet. 2000;9(18):2743-2750.

24. Veland IR, Awan A, Pedersen LB, Yoder BK, Christensen ST. Primary cilia and signaling pathways in mammalian development, health and disease. Nephron Physiol. 2009;111(3):39-53.

25. Pazour GJ, San Agustin JT, Follit JA, Rosenbaum JL, Witman GB. Polycystin-2 localizes to kidney cilia and the ciliary level is elevated in orpk mice with polycystic kidney disease. Curr Biol. 2002;12(11):R378-R380.

26. Yoder BK, Hou X, Guay-Woodford LM. The polycystic kidney disease proteins, polycystin-1, polycystin-2, polaris, and cystin, are co-localized in renal cilia. J Am Soc Nephrol. 2002;13(10):2508-2516.

27. Hildebrandt F, Benzing T, Katsanis N. Ciliopathies. N Engl J Med. 2011;364(16):1533-1543.

28. Sun Z, Amsterdam A, Pazour GJ, Cole DG, Miller MS, Hopkins N. A genetic screen in zebrafish identifies cilia genes as a principal cause of cystic kidney. Development. 2004;131(16):4085-4093.

29. Lin F, et al. Kidney-specific inactivation of the KIF3A subunit of kinesin-II inhibits renal ciliogenesis and produces polycystic kidney disease. Proc Natl Acad Sci U S A. 2003;100(9):5286-5291.

30. Davenport JR, et al. Disruption of intraflagellar transport in adult mice leads to obesity and slow-onset cystic kidney disease. Curr Biol. 2007;17(18):1586-1594.

31. Jonassen JA, San AJ, Follit JA, Pazour GJ. Deletion of IFT20 in the mouse kidney causes misorientation of the mitotic spindle and cystic kidney disease. J Cell Biol. 2008;183(3):377-384.

32. Ma M, Tian X, Igarashi P, Pazour GJ, Somlo S. Loss of cilia suppresses cyst growth in genetic models of autosomal dominant polycystic kidney disease. Nat Genet. 2013;45(9):1004-1012.

33. Nims N, Vassmer D, Maser RL. Transmembrane domain analysis of polycystin-1, the product of the polycystic kidney disease-1 (PKD1) gene: evidence for 11 membrane-spanning domains. Biochemistry. 2003;42(44):13035-13048. 
34. Hughes J, et al. The polycystic kidney disease 1 (PKD1) gene encodes a novel protein with multiple cell recognition domains. Nat Genet. 1995;10(2):151-160.

35. [No authors listed]. Polycystic kidney disease: the complete structure of the PKD1 gene and its protein. The International Polycystic Kidney Disease Consortium. Cell. 1995;81(2):289-298.

36. Sandford R, et al. Comparative analysis of the polycystic kidney disease 1 (PKD1) gene reveals an integral membrane glycoprotein with multiple evolutionary conserved domains. Hum Mol Genet. 1997;6(9):1483-1489.

37. Moy GW, Mendoza LM, Schulz JR, Swanson WJ, Glabe CG, Vacquier VD. The sea urchin sperm receptor for egg jelly is a modular protein with extensive homology to the human polycystic kidney disease protein, PKD1. J Cell Biol. 1996;133(4):809-817.

38. Schroder S, Fraternali F, Quan X, Scott D, Qian $\mathrm{F}$, Pfuhl $\mathrm{M}$. When a module is not a domain: the case of the REJ module and the redefinition of the architecture of polycystin-1. Biochem J. 2011;435(3):651-660.

39. Arac D, et al. A novel evolutionarily conserved domain of cell-adhesion GPCRs mediates autoproteolysis. EMBO J. 2012;31(6):1364-1378.

40. Ponting CP, Hofmann K, Bork P. A latrophilin/ CL-1-like GPS domain in polycystin-1. Curr Biol. 1999;9(16):R585-R5R8.

41. Qian F, et al. Cleavage of polycystin-1 requires the receptor for egg jelly domain and is disrupted by human autosomal-dominant polycystic kidney disease 1-associated mutations. Proc Natl Acad Sci U S A. 2002;99(26):16981-16986.

42. Wei W, Hackmann K, Xu H, Germino G, Qian F. Characterization of cis-autoproteolysis of polycystin-1, the product of human polycystic kidney disease 1 gene. J Biol Chem. 2007;282(30):21729-21737.

43. Li A, Tian X, Sung SW, Somlo S. Identification of two novel polycystic kidney disease-1-like genes in human and mouse genomes. Genomics. 2003;81(6):596-608.

44. Butscheid Y, Chubanov V, Steger K, Meyer D, Dietrich A, Gudermann T. Polycystic kidney disease and receptor for egg jelly is a plasma membrane protein of mouse sperm head. Mol Reprod Dev. 2006;73(3):350-360.

45. Galindo BE, Moy GW, Vacquier VD. A third sea urchin sperm receptor for egg jelly module protein, suREJ2, concentrates in the plasma membrane over the sperm mitochondrion. Dev Growth Differ. 2004;46(1):53-60.

46. PKD Foundation. Autosomal Dominant Polycystic Kidney Disease Mutation Database: PKDB. http://pkdb.mayo.edu/. PKD Foundation Web site. Accessed October 1, 2014.

47. Hopp K, et al. Functional polycystin-1 dosage governs autosomal dominant polycystic kidney disease severity. J Clin Invest. 2012;122(11):4257-4273.

48. Cornec-Le Gall E, et al. Type of PKD1 mutation influences renal outcome in ADPKD. J Am Soc
Nephrol. 2013;24(6):1006-1013.

49. Cai Y, et al. Calcium dependence of polycystin-2 channel activity is modulated by phosphorylation at Ser812. J Biol Chem. 2004;279(19):19987-19995.

50. Kurbegovic A, et al. Novel functional complexity of polycystin-1 by GPS cleavage in vivo: role in polycystic kidney disease. Mol Cell Biol. 2014;34(17):3341-3353.

51. Cai Y, et al. Identification and characterization of polycystin-2, the PKD2 gene product. J Biol Chem. 1999;274(40):28557-28565.

52. Yu Y, et al. Structural and molecular basis of the assembly of the TRPP2/PKD1 complex. Proc Natl Acad Sci U S A. 2009;106(28):11558-11563.

53. Casuscelli J, et al. Analysis of the cytoplasmic interaction between polycystin- 1 and polycystin-2. Am J Physiol Renal Physiol. 2009;297(5):F1310-F13F5.

54. Bycroft M, et al. The structure of a PKD domain from polycystin-1: implications for polycystic kidney disease. EMBO J. 1999;18(2):297-305.

55. Veldhuisen B, et al. A spectrum of mutations in the second gene for autosomal dominant polycystic kidney disease (PKD2). Am J Hum Genet. 1997;61(3):547-555

56. Reynolds DM, et al. Aberrant splicing in the PKD2 gene as a cause of polycysitic kidney disease. J Am Soc Nephrol. 1999;10(11):2342-2351.

57. Lalioti M, Heath J. A new method for generating point mutations in bacterial artificial chromosomes by homologous recombination in Escherichia coli. Nucleic Acids Res. 2001;29(3):E14.

58. Lalioti MD, et al. Wnk4 controls blood pressure and potassium homeostasis via regulation of mass and activity of the distal convoluted tubule. Nat Genet. 2006;38(10):1124-1132.

59. Chauvet V, et al. Mechanical stimuli induce cleavage and nuclear translocation of the polycystin-1 C terminus. JClin Invest. 2004;114(10):1433-1443.

60. Pritchard L, et al. A human PKD1 transgene generates functional polycystin- 1 in mice and is associated with a cystic phenotype. Hum Mol Genet. 2000;9(18):2617-2627.

61. Thivierge C, Kurbegovic A, Couillard M, Guillaume R, Cote O, Trudel M. Overexpression of PKD1 causes polycystic kidney disease. Mol Cell Biol. 2006;26(4):1538-1548.

62. Lohning C, Nowicka U, Frischauf AM. The mouse homolog of PKD1: sequence analysis and alternative splicing. Mamm Genome. 1997;8(5):307-311.

63. Geng L, et al. Distribution and developmentally regulated expression of murine polycystin. Am J Physiol. 1997;272(4 pt 2):F451-F4F9.

64. Wodarczyk C, Rowe I, Chiaravalli M, Pema M, Qian F, Boletta A. A novel mouse model reveals that polycystin-1 deficiency in ependyma and choroid plexus results in dysfunctional cilia and hydrocephalus. PLoS One. 2009;4(9):e7137.

65. Shibazaki S, et al. Cyst formation and activation of the extracellular regulated kinase pathway after kidney specific inactivation of Pkd1. Hum Mol Genet. 2008;17(11):1505-1516.

66. Karcher C, et al. Lack of a laterality phenotype in
Pkd1 knock-out embryos correlates with absence of polycystin-1 in nodal cilia. Differentiation. 2005;73(8):425-432.

67. Barr MM, DeModena J, Braun D, Nguyen CQ, Hall DH, Sternberg PW. The Caenorhabditis elegans autosomal dominant polycystic kidney disease gene homologs lov- 1 and pkd- 2 act in the same pathway. Curr Biol. 2001;11(17):1341-1346.

68. Fedeles SV, Gallagher AR, Somlo S. Polycystin-1: a master regulator of intersecting cystic pathways. Trends Mol Med.2014;20(5):251-260.

69. Ramsey BW, et al. A CFTR potentiator in patients with cystic fibrosis and the G551D mutation. N EnglJMed. 2011;365(18):1663-1672.

70. Sanders CR, Myers JK. Disease-related misassembly of membrane proteins. Annu Rev Biophys Biomol Struct. 2004;33:25-51.

71. Cohen FE, Kelly JW. Therapeutic approaches to protein-misfolding diseases. Nature. 2003;426(6968):905-909.

72. Kuznetsov G, Nigam SK. Folding of secretory and membrane proteins. N EnglJMed. 1998;339(23):1688-1695.

73. Yu S, et al. Essential role of cleavage of Polycystin-1 at G protein-coupled receptor proteolytic site for kidney tubular structure. Proc Natl Acad Sci US A. 2007;104(47):18688-18693.

74. Giamarchi A, et al. A polycystin-2 (TRPP2) dimerization domain essential for the function of heteromeric polycystin complexes. EMBO J. 2010;29(7):1176-1191.

75. DeCaen PG, Delling M, Vien TN, Clapham DE. Direct recording and molecular identification of the calcium channel of primary cilia. Nature. 2013;504(7479):315-318.

76. Delling M, DeCaen PG, Doerner JF, Febvay S, Clapham DE. Primary cilia are specialized calcium signalling organelles. Nature. 2013;504(7479):311-314.

77. Mu TW, et al. Chemical and biological approaches synergize to ameliorate protein-folding diseases. Cell. 2008;134(5):769-781.

78. Kurbegovic A, Côté O, Couillard M, Ward CJ, Harris PC, Trudel M. Pkd1 transgenic mice: adult model of polycystic kidney disease with extrarenal and renal phenotypes. Hum Mol Genet. 2010;19(7):1174-1189.

79. Wang $\mathrm{N}$, et al. A PEST sequence in ABCA1 regulates degradation by calpain protease and stabilization of ABCA1 by apoA-I. J Clin Invest. 2003;111(1):99-107.

80. Choi YH, et al. Polycystin-2 and phosphodiesterase $4 \mathrm{C}$ are components of a ciliary A-kinase anchoring protein complex that is disrupted in cystic kidney diseases. Proc Natl Acad Sci U S A. 2011;108(26):10679-10684.

81. Ballester M, Castello A, Ibanez E, Sanchez A, Folch JM. Real-time quantitative PCR-based system for determining transgene copy number in transgenic animals. BioTechniques. 2004;37(4):610-613.

82. Grimm DH, et al. Polycystin-1 distribution is modulated by polycystin- 2 expression in mammalian cells. J Biol Chem. 2003;278(38):36786-36793. 